\title{
Chaetopteryx bucari sp. $n$. , a new species from the Chaetopteryx rugulosa group from Croatia (Insecta, Trichoptera, Limnephilidae) with molecular, taxonomic and ecological notes on the group
}

\author{
Mladen Kučinićl,†, Ildikó Szivák ${ }^{2, \ddagger}$, Steffen U. Pauls,\$, \\ Miklós Bálint ${ }^{3,1}$, Antun Delić, ${ }^{4, \pi}$, Ivan Vučkovićs ${ }^{5, \#}$
}

I Department of Biology (Group for Systematic Zoology \& Entomology), Faculty of Science, University of Zagreb, Rooseveltov trg 6, 10000 Zagreb, Croatia 2 Balaton Limnological Institute, Centre for Ecological Research, Hungarian Academy of Science, H-8237 Tihany, Klebelsberg Kuno u. 3, Hungary 3 Biodiversity and Climate Research Centre (BiK-F), Senckenberg Gesellschaft für Naturforschung, Senckenberganlage 25, 60325 Frankfurt am Main, Germany 4 Faculty of Ecudation, Department in Petrinja, University of Zagreb, Matice Hrvatske 12, 44250, Petrinja, Croatia 5 Elektroprojekt d.d., Civil and Architectural Engineering Department, Alexandera von Humboldta 4, 10000 Zagreb, Croatia

† http://zoobank.org/62E138E2-169A-4680-B96E-232BC4FB9C4B

¥ttp://zoobank.org/3ACAC586-1156-4EE3-9B52-0B40966801DA

§ http://zoobank.org/16F1195D-04AE-4C13-87E9-017C632969E0

| http://zoobank.org/9131E7ED-B036-44C3-B996-78B8C317B82E

I http://zoobank.org/BE7462E8-4584-4F0B-A50F-DC7BC696809C

\# http://zoobank.org/C38ECB62-BDA7-4A53-84C3-166C13856AF9

Corresponding author: Mladen Kučinić (kucinic@zg.biol.pmf.hr)

Academic editor: R. Holzenthal | Received 23 December 2012 | Accepted 27 June 2013 | Published 31 July 2013

http://zoobank.org/2ABD421F-E3D0-4D8B-A03E-AD67EE489FEE

Citation: Kučinić M, Szivák I, Pauls SU, Bálint M, Delić A, Vučković I (2013) Chaetopteryx bucari sp. n., a new species from the Chaetopteryx rugulosa group from Croatia (Insecta, Trichoptera, Limnephilidae) with molecular, taxonomic and ecological notes on the group. ZooKeys 320: 1-28. doi: 10.3897/zookeys.320.4565

\begin{abstract}
We describe a new autumnal caddisfly species Chaetopteryx bucari sp. n. from 8 localities in the Banovina region of Croatia. We also present molecular, taxonomic and ecological notes (emergence, sex ratio and seasonal dynamics) on the new species and discuss the distribution of Chaetopteryx species in general and the C. rugulosa group in particular. Based on Bayesian phylogenetic analysis C. rugulosa schmidi was sepa-
\end{abstract}

Copyright Mladen Kučinić et al. This is an open access article distributed under the terms of the Creative Commons Attribution License 3.0 (CC-BY), which permits unrestricted use, distribution, and reproduction in any medium, provided the original author and source are credited. 
rated from the clade containing the other subspecies of $C$. rugulosa. Thus the subspecies $C$. $r$. schmidi is here raised to species level, C. schmidi, as it was described originally. We further present distribution data on rare species in the genus Chaetopteryx in Croatia.

\section{Keywords}

Chaetopteryx, aquatic insects, new species, distribution, Croatia

\section{Introduction}

The genus Chaetopteryx belongs to a small number of caddisfly genera with adults that are adapted to low air temperatures and emerge in autumn or winter, mostly from October-January. The larvae of most species live in small headwater streams and springs. This genus is distributed in Europe and parts of Asia (e.g., Asia Minor, Iran) (Malicky 2004, Lodovici and Valle 2007, Sipahiler 2010). In Europe, Chaetopteryx comprises 25 species (Malicky 2004, Lodovici and Valle 2007, Oláh 2011a, 2011b). A particularly interesting species group in the genus is the Chaetopteryx rugulosa group. This radiation consists of 6 species and 3 subspecies: Chaetopteryx rugulosa rugulosa Kolenati, 1848; Chaetopteryx rugulosa mecsekensis Nógrádi, 1986; Chaetopteryx rugulosa noricum Malicky, 1976; Chaetopteryx rugulosa schmidi Botosaneanu, 1957; Chaetopteryx clara McLachlan, 1876; Chaetopteryx euganea Moretti \& Malicky, 1986; Chaetopteryx goricensis Malicky and Krušnik, 1986; Chaetopteryx irenae Krušnik \& Malicky, 1986 and Chaetopteryx marinkovicae Malicky and Krušnik, 1988 (Malicky 2004).

Four years ago we started systematically collecting adults of the genus Chaetopteryx, including members of the C. rugulosa group in Croatia. This paper has 2 main objectives, first to present and describe a new species from the $C$. rugulosa group found in Croatia, and second to present new molecular, taxonomic, distributional, and ecological information on the C. rugulosa group.

\section{Material and methods}

Fieldwork. We collected specimens of Chaetopteryx including C. rugulosa group species in the continental (central Croatia, Banovina, Hrvatsko zagorje, Kordun, Slavonia), mountain (Gorski kotar, Lika regions) and Mediterranean (Istria and Dalmatia) regions of Croatia. Collecting methods included the use of entomological nets and handpicking specimens from walls of small buildings or wells, or from the riparian vegetation near springs and headwater streams. In one spring (Pecki spring, Banovina region) (Table 1) we installed 5 pyramid-type emergence traps in 2010 and 2011 to investigate the emergence dynamics of caddisflies (Figure 1). This investigation is part of a multi-year study on emergence dynamics of aquatic insects in springs and other aquatic habitats in Croatia and the Dinaric karst of the Balkan Peninsula (Bosnia and Herzegovina) (Kučinić 2002, Previšić et al. 2007, Ivković et al. 2011, Semnički et al. 
Table I. Localities where Chaetopteryx bucari, sp. n., was collected, including habitat type, elevation (m a.s.l.), and geographic coordinates.

\begin{tabular}{l|c|c|c}
\hline \multicolumn{1}{c|}{ Location } & Character of location & Altitude $(\mathbf{m})$ & Geographic coordinates \\
\hline Bijele stijene & wellspring and stream & 144 & $45^{\circ} 25^{\prime} 23^{\prime \prime} \mathrm{N}, 16^{\circ} 13^{\prime} 23^{\prime \prime} \mathrm{E}$ \\
\hline Gore & wellspring & 165 & $45^{\circ} 24^{\prime} 21^{\prime \prime N}, 16^{\circ} 14^{\prime} 22^{\prime \prime} \mathrm{E}$ \\
\hline Hrvatski Čuntić & stream & 159 & $45^{\circ} 21^{\prime} 28^{\prime \prime N}, 16^{\circ} 17^{\prime} 04^{\prime \prime} \mathrm{E}$ \\
\hline Marića točak & wellspring & 163 & $45^{\circ} 21^{\prime} 29^{\prime \prime} \mathrm{N}, 16^{\circ} 17^{\prime} 03^{\prime \prime} \mathrm{E}$ \\
\hline Pašino vrelo & spring & 185 & $45^{\circ} 17^{\prime} 16^{\prime \prime N}, 16^{\circ} 25^{\prime} 13^{\prime \prime} \mathrm{E}$ \\
\hline Pecki & spring & 161 & $45^{\circ} 23^{\prime} 50^{\prime \prime} \mathrm{N}, 16^{\circ} 14^{\prime} 40^{\prime \prime} \mathrm{E}$ \\
\hline Slabinja & wellspring & 104 & $45^{\circ} 13^{\prime} 05^{\prime \prime} \mathrm{N}, 16^{\circ} 37^{\prime} 52^{\prime \prime} \mathrm{E}$ \\
\hline Varoški bunar & wellspring & 130 & $45^{\circ} 13^{\prime} 34^{\prime \prime N}, 16^{\circ} 33^{\prime} 12^{\prime \prime} \mathrm{E}$ \\
\hline
\end{tabular}

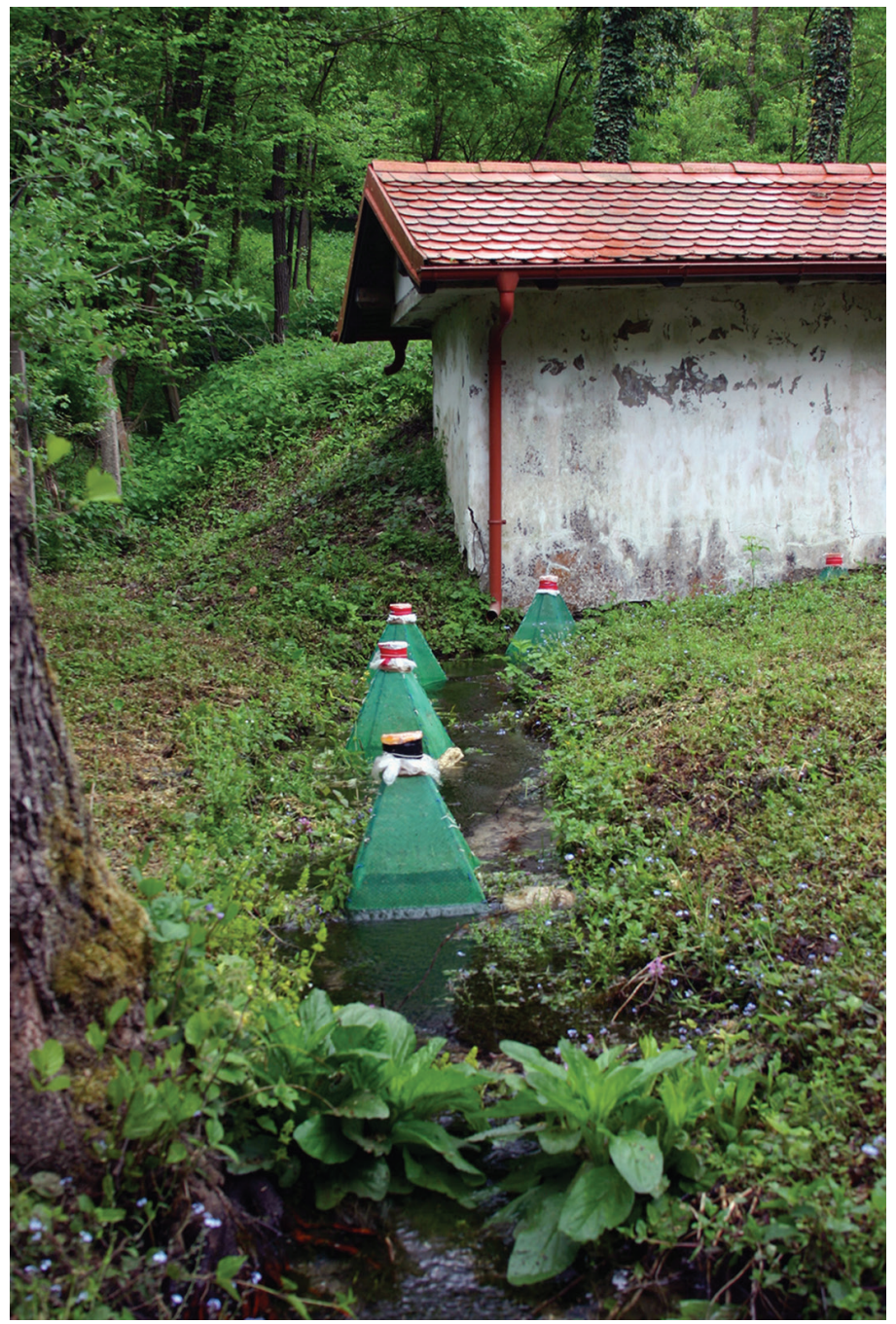

Figure I. Type locality of Chaetopteryx bucari, sp. n., showing pyramid-type emergence traps, Pecki spring, Croatia. 
2011, 2012, M. Kučinić unpublished data). The emergence trapping methodology was presented in detail by Kučinić (2002) and Previšić et al. (2007).

In pyramid-type emergence traps caddisflies were collected in $1 \%$ formaldehyde and thereafter stored in $80 \%$ alcohol. All other collected specimens were stored directly in $80 \%$ or $96 \%$ alcohol. All specimens were deposited in the collections of the first and second authors. The holotype is deposited in the Croatian Natural History Museum in Zagreb.

Laboratory work. For the phylogenetic analysis we compiled mtCOI DNA sequence data for 103 specimens from the C. rugulosa group (Table 2). We also sequenced several outgroup taxa of varying putative phylogenetic depths including congeneric species (e.g., Chaetopteryx gessneri McLachlan, 1876, Chaetopteryx fusca Brauer, 1857, Chaetopteryx major McLachalan, 1876, Chaetopteryx villosa (Fabricius, 1798)), other members of the tribe Chaetopterygini (Chaetopterygopsis maclachlani (Stein, 1874)), other members of the subfamily Limnephilinae (Limnephilus centralis Curtis, 1834), and members of a different subfamily of Limnephilidae (e.g. Metanoea rhaetica Schmid, 1955, Drusus alpinus (Meyer-Dür, 1875), Drusus rectus McLachlan, 1868).

Systematic presentation follows Morse (2013). The terminology and morphological assessment of the C. rugulosa group follows Malicky et al. (1986), Malicky and Krušnik (1988), Urbanič and Krušnik (2003), Botosaneanu and Giudicelli (2004), Holzenthal et al. (2007), Oláh (2011a), and Vučković et al. (2011). Comparative assessments of morphological features of $C$. bucari were based on the other specimens collected in Croatia (C. r. rugulosa, C. marinkovicae) or based on literature (e.g., C. r. schmidi, C. r. mecsekensis, Malicky et al. 1986, Malicky 2004). Morphological features of genitalia of C. bucari were analysed from 84 specimens ( 40 males and 44 females).

The mitochondrial COI barcodes were generated at the Canadian Centre for DNA Barcoding, University of Guelph, Canada. Standard barcoding protocols for DNA extraction (Ivanova et al. 2006), PCR amplification and COI sequencing (Hajibabaei et al. 2005, de Waard et al. 2008) were used. Full-length COI-5P DNA barcodes were amplified using C_LepFolF/C_LepFolR (Folmer et al. 1994, Hajibabaei et al. 2006) and LCO1490/HCO2198 (Folmer et al. 1994) primer sets. COI barcodes and detailed specimen information can be found in the Barcode of Life Data Systems (BOLD; http://www.boldsystems.org/) (Ratnasingham and Hebert 2007) within the project "Chaetopteryx of Europe." Unpublished COI barcodes of additional Chaetopteryx outgroups were provided by Karl Kjer, Rutgers University, USA (Table 2). The sequence of Limnephilus centralis Curtis, 1834 was taken from Malm and Johanson (2011) (Table 2).

Phylogenetic analysis. Sequences were edited manually and aligned using the program Geneious 5.4 (Drummond et al. 2011). The final alignment was 617 base pairs (bp) long. Bayesian phylogenetic analyses were performed using the Markov chain Monte Carlo method (B/MCMC) using MrBayes 3.2 (Buckley et al. 2002, Ronquist and Huelsenbeck 2003). We selected the best-fitting models of DNA substitution using Akaike information criterion (AIC) implemented in jModelTest 0.1.1 (Guindon and Gascuel 2003, Posada 2008). jModelTest indicated a general time re- 
Table 2. List of species included in the DNA analysis (mtCOI sequences). Localities are given with country code, locality/specimen data, and collection date.

\begin{tabular}{|c|c|c|c|c|}
\hline Species name & Locality & Specimen ID & $\begin{array}{c}\text { Accession } \\
\text { number }\end{array}$ & $\begin{array}{l}\text { Collectors/ } \\
\text { Source }\end{array}$ \\
\hline Chaetopteryx aproka & $\begin{array}{l}\text { ROU, Ignis Mts., springs near } \\
\text { Desesti-Statiunea Izvoare, } \\
21.10 .2010\end{array}$ & CAxJC0101 & HE858253 & $\begin{array}{l}\text { Ecsedi, Olah } \\
\text { \& Szivak }\end{array}$ \\
\hline Chaetopteryx aproka & $\begin{array}{l}\text { ROU, Ignis Mts., springs near } \\
\text { Desesti-Statiunea Izvoare, } \\
21.10 .2010\end{array}$ & CAxJC0102 & HE858254 & $\begin{array}{l}\text { Ecsedi, Olah } \\
\& \text { Szivak }\end{array}$ \\
\hline Chaetopteryx aproka & $\begin{array}{l}\text { ROU, Ignis Mts., springs near } \\
\text { Desesti-Statiunea Izvoare, } \\
21.10 .2010\end{array}$ & CAxJC0103 & HE858255 & $\begin{array}{l}\text { Ecsedi, Olah } \\
\text { \& Szivak }\end{array}$ \\
\hline $\begin{array}{l}\text { Chaetopteryx } \\
\text { bosniaca }\end{array}$ & $\begin{array}{l}\text { BIH, Livno, Sturba river, } \\
08.11 .2009\end{array}$ & CBxED0101 & & $\begin{array}{l}\text { Kučinić, } \\
\text { Delić \& } \\
\text { Mihoci }\end{array}$ \\
\hline $\begin{array}{l}\text { Chaetopteryx } \\
\text { bosniaca }\end{array}$ & $\begin{array}{l}\text { BIH, Livno, Sturba river, } \\
\text { 08.11.2009 }\end{array}$ & CBxED0102 & & $\begin{array}{l}\text { Kučinić, } \\
\text { Delić \& } \\
\text { Mihoci }\end{array}$ \\
\hline $\begin{array}{l}\text { Chaetopteryx } \\
\text { bosniaca }\end{array}$ & $\begin{array}{l}\text { BIH, Livno, Sturba river, } \\
\text { 08.11.2009 }\end{array}$ & CBxED0103 & & $\begin{array}{l}\text { Kučinić, } \\
\text { Delić \& } \\
\text { Mihoci }\end{array}$ \\
\hline $\begin{array}{l}\text { Chaetopteryx } \\
\text { bosniaca }\end{array}$ & $\begin{array}{l}\text { BIH, Livno, Sturba river, } \\
\text { 08.11.2009 }\end{array}$ & CBxED0104 & & $\begin{array}{l}\text { Kučinić, } \\
\text { Delić \& } \\
\text { Mihoci }\end{array}$ \\
\hline $\begin{array}{l}\text { Chaetopteryx } \\
\text { bosniaca }\end{array}$ & $\begin{array}{l}\text { BIH, Livno, Sturba river, } \\
\text { 08.11.2009 }\end{array}$ & CBxED0105 & & $\begin{array}{l}\text { Kučinić, } \\
\text { Delić \& } \\
\text { Mihoci }\end{array}$ \\
\hline Chaetopteryx clara & $\begin{array}{l}\text { SLO, Ljubljana, Mostec park, } \\
\text { Przanec stream, 06.12.2009 }\end{array}$ & CCxEA0101 & JF891164 & $\begin{array}{l}\text { Dery \& } \\
\text { Szivak }\end{array}$ \\
\hline Chaetopteryx clara & \begin{tabular}{|l} 
SLO, Ljubljana, Mostec park, \\
Przanec stream, 06.12.2009
\end{tabular} & CCxEA0102 & JF891165 & $\begin{array}{l}\text { Dery \& } \\
\text { Szivak }\end{array}$ \\
\hline Chaetopteryx clara & \begin{tabular}{|l} 
SLO, Ljubljana, Mostec park, \\
Przanec stream, 06.12.2009
\end{tabular} & CCxEA0103 & JF891166 & $\begin{array}{l}\text { Dery \& } \\
\text { Szivak }\end{array}$ \\
\hline Chaetopteryx clara & $\begin{array}{l}\text { SLO, Ljubljana, Mostec park, } \\
\text { Przanec stream, 06.12.2009 }\end{array}$ & CCxEA0104 & JF891167 & $\begin{array}{l}\text { Dery \& } \\
\text { Szivak }\end{array}$ \\
\hline Chaetopteryx clara & $\begin{array}{l}\text { SLO, Ljubljana, Mostec park, } \\
\text { Przanec stream, 06.12.2009 }\end{array}$ & CCxEA0105 & JF891168 & $\begin{array}{l}\text { Dery \& } \\
\text { Szivak }\end{array}$ \\
\hline $\begin{array}{l}\text { Chaetopteryx } \\
\text { goricensis }\end{array}$ & $\begin{array}{l}\text { SLO, spring of Lokavscek stream } \\
\text { near Predmeja, 06.12.2009 }\end{array}$ & CGREG0101 & JF891159 & $\begin{array}{l}\text { Dery \& } \\
\text { Szivak }\end{array}$ \\
\hline $\begin{array}{l}\text { Chaetopteryx } \\
\text { goricensis }\end{array}$ & $\begin{array}{l}\text { SLO, spring of Lokavscek stream } \\
\text { near Predmeja, 06.12.2009 }\end{array}$ & CGREG0102 & JF891160 & $\begin{array}{l}\text { Dery \& } \\
\text { Szivak }\end{array}$ \\
\hline $\begin{array}{l}\text { Chaetopteryx } \\
\text { goricensis }\end{array}$ & $\begin{array}{l}\text { SLO, spring of Lokavscek stream } \\
\text { near Predmeja, 06.12.2009 }\end{array}$ & CGREG0103 & JF891161 & $\begin{array}{l}\text { Dery \& } \\
\text { Szivak }\end{array}$ \\
\hline $\begin{array}{l}\text { Chaetopteryx } \\
\text { goricensis }\end{array}$ & $\begin{array}{l}\text { SLO, spring of Lokavscek stream } \\
\text { near Predmeja, 06.12.2009 }\end{array}$ & CGREG0104 & JF891162 & $\begin{array}{l}\text { Dery \& } \\
\text { Szivak }\end{array}$ \\
\hline $\begin{array}{l}\text { Chaetopteryx } \\
\text { goricensis }\end{array}$ & $\begin{array}{l}\text { SLO, spring of Lokavscek stream } \\
\text { near Predmeja, 06.12.2009 }\end{array}$ & CGREG0105 & JF891163 & $\begin{array}{l}\text { Dery \& } \\
\text { Szivak }\end{array}$ \\
\hline $\begin{array}{l}\text { Chaetopteryx } \\
\text { goricensis }\end{array}$ & $\begin{array}{l}\text { SLO, spring near Čekovnik } \\
\text { (Hlevise), 05.12.2009 }\end{array}$ & CGREG0201 & JF891154 & $\begin{array}{l}\text { Dery \& } \\
\text { Szivak }\end{array}$ \\
\hline $\begin{array}{l}\text { Chaetopteryx } \\
\text { goricensis }\end{array}$ & $\begin{array}{l}\text { SLO, spring near Čekovnik } \\
\text { (Blask), 05.12.2009 }\end{array}$ & CGREG0301 & JF891155 & $\begin{array}{l}\text { Dery \& } \\
\text { Szivak }\end{array}$ \\
\hline $\begin{array}{l}\text { Chaetopteryx } \\
\text { goricensis }\end{array}$ & $\begin{array}{l}\text { SLO, spring near Čekovnik } \\
\text { (Blask), 05.12.2009 }\end{array}$ & CGREG0302 & JF891156 & $\begin{array}{l}\text { Dery \& } \\
\text { Szivak }\end{array}$ \\
\hline $\begin{array}{l}\text { Chaetopteryx } \\
\text { goricensis }\end{array}$ & $\begin{array}{l}\text { SLO, spring near Čekovnik } \\
\text { (Blask), 05.12.2009 }\end{array}$ & CGREG0303 & JF891157 & $\begin{array}{l}\text { Dery \& } \\
\text { Szivak }\end{array}$ \\
\hline
\end{tabular}




\begin{tabular}{|c|c|c|c|c|}
\hline Species name & Locality & Specimen ID & $\begin{array}{c}\text { Accession } \\
\text { number }\end{array}$ & $\begin{array}{c}\text { Collectors/ } \\
\text { Source }\end{array}$ \\
\hline $\begin{array}{l}\text { Chaetopteryx } \\
\text { goricensis }\end{array}$ & $\begin{array}{l}\text { SLO, spring near Čekovnik } \\
\text { (Blask), 05.12.2009 }\end{array}$ & CGREG0304 & JF891158 & $\begin{array}{l}\text { Dery \& } \\
\text { Szivak }\end{array}$ \\
\hline Chaetopteryx irenae & $\begin{array}{l}\text { SLO, Susica stream near Misliče, } \\
06.12 .2009\end{array}$ & CIxEI0101 & JF891169 & $\begin{array}{l}\text { Dery \& } \\
\text { Szivak }\end{array}$ \\
\hline Chaetopteryx irenae & $\begin{array}{l}\text { SLO, Susica stream near Misliče, } \\
06.12 .2009\end{array}$ & CIxEI0102 & JF891170 & $\begin{array}{l}\text { Dery \& } \\
\text { Szivak }\end{array}$ \\
\hline Chaetopteryx irenae & $\begin{array}{l}\text { SLO, Misliče, Susica stream, } \\
\text { 06.12.2009 }\end{array}$ & CIxEI0103 & JF891171 & $\begin{array}{l}\text { Dery \& } \\
\text { Szivak }\end{array}$ \\
\hline Chaetopteryx irenae & $\begin{array}{l}\text { SLO, Misliče, Susica stream, } \\
\text { 06.12.2009 }\end{array}$ & CIxEI0104 & JF891172 & $\begin{array}{l}\text { Dery \& } \\
\text { Szivak }\end{array}$ \\
\hline Chaetopteryx irenae & $\begin{array}{l}\text { SLO, Misliče, Susica stream, } \\
\text { 06.12.2009 }\end{array}$ & CIxEI0105 & JF891173 & $\begin{array}{l}\text { Dery \& } \\
\text { Szivak }\end{array}$ \\
\hline Chaetopteryx major & $\begin{array}{l}\text { HUN, Mecsek Mts., Vár valley, } \\
\text { Pásztor spring 05.11.2010 }\end{array}$ & CMJKB0101 & JF891233 & \begin{tabular}{|l} 
Olah, \\
Szivak \& \\
Uherkovich \\
\end{tabular} \\
\hline Chaetopteryx major & $\begin{array}{l}\text { HUN, Mecsek Mts., Vár valley, } \\
\text { Pásztor spring 05.11.2010 }\end{array}$ & CMJKB0102 & HE858256 & $\begin{array}{l}\text { Olah, } \\
\text { Szivak \& } \\
\text { Uherkovich }\end{array}$ \\
\hline Chaetopteryx major & $\begin{array}{l}\text { HUN, Mecsek Mts., Vár valley, } \\
\text { Pásztor spring 05.11.2010 }\end{array}$ & CMJKB0103 & HE858257 & \begin{tabular}{|l} 
Olah, \\
Szivak \& \\
Uherkovich \\
\end{tabular} \\
\hline Chaetopteryx major & $\begin{array}{l}\text { HUN, Mecsek Mts., Vár valley, } \\
\text { Pásztor spring 05.11.2010 }\end{array}$ & CMJKB0104 & HE858258 & \begin{tabular}{|l} 
Olah, \\
Szivak \& \\
Uherkovich \\
\end{tabular} \\
\hline Chaetopteryx major & $\begin{array}{l}\text { AUT, valley Hottmannsgraben, } \\
\text { Unteraspang (Aspang Markt) } \\
\text { 19.11.2009 }\end{array}$ & CMJDJ0101 & JF891234 & $\begin{array}{l}\text { Dery \& } \\
\text { Szivak }\end{array}$ \\
\hline $\begin{array}{l}\text { Chaetopteryx } \\
\text { marinkovicae }\end{array}$ & CRO, Kompanj, 14.11.2009 & CMREI0101 & JF891174 & $\begin{array}{l}\text { Kučinić \& } \\
\text { Vučković }\end{array}$ \\
\hline $\begin{array}{l}\text { Chaetopteryx } \\
\text { marinkovicae }\end{array}$ & CRO, Kompanj, 14.11.2009 & CMREI0102 & JF891175 & $\begin{array}{l}\text { Kučinić \& } \\
\text { Vučković }\end{array}$ \\
\hline $\begin{array}{l}\text { Chaetopteryx } \\
\text { marinkovicae }\end{array}$ & CRO, Kompanj, 14.11.2009 & CMREI0103 & JF891176 & $\begin{array}{l}\text { Kučinić \& } \\
\text { Vučković }\end{array}$ \\
\hline $\begin{array}{l}\text { Chaetopteryx } \\
\text { marinkovicae }\end{array}$ & CRO, Kompanj, 14.11.2009 & CMREI0104 & JF891177 & $\begin{array}{l}\text { Kučinić \& } \\
\text { Vučković }\end{array}$ \\
\hline $\begin{array}{l}\text { Chaetopteryx } \\
\text { marinkovicae }\end{array}$ & CRO, Kompanj, 14.11.2009 & CMREI0105 & JF891178 & $\begin{array}{l}\text { Kučinić \& } \\
\text { Vučković }\end{array}$ \\
\hline $\begin{array}{l}\text { Chaetopteryx } \\
\text { rugulosa mecsekensis }\end{array}$ & $\begin{array}{l}\text { HUN, Mecsek Mts., Nagy-Mély } \\
\text { valley, Kánya spring, 14.11.2009 }\end{array}$ & CRMKB0101 & JF891179 & Szivak \\
\hline $\begin{array}{l}\text { Chaetopteryx } \\
\text { rugulosa mecsekensis }\end{array}$ & $\begin{array}{l}\text { HUN, Mecsek Mts., Vár valley, } \\
\text { Pásztor spring, 06.11.2009 }\end{array}$ & CRMKB0201 & JF891180 & $\begin{array}{l}\text { Szivak \& } \\
\text { Uherkovich }\end{array}$ \\
\hline $\begin{array}{l}\text { Chaetopteryx } \\
\text { rugulosa mecsekensis }\end{array}$ & $\begin{array}{l}\text { HUN, Mecsek Mts., Melegmányi } \\
\text { valley, Mésztufa spring, } \\
14.11 .2009\end{array}$ & CRMKB0301 & JF891203 & Szivak \\
\hline $\begin{array}{l}\text { Chaetopteryx } \\
\text { rugulosa mecsekensis }\end{array}$ & $\begin{array}{l}\text { HUN, Mecsek Mts., Vár valley, } \\
\text { Iharos spring, 06.11.2009 }\end{array}$ & CRMKB0401 & JF891204 & Szivak \\
\hline $\begin{array}{l}\text { Chaetopteryx } \\
\text { rugulosa noricum }\end{array}$ & $\begin{array}{l}\text { AUT, Saualpe, Klieningbach } \\
\text { stream near Kliening, 21.11.2009 }\end{array}$ & CRNDI0101 & JF891187 & $\begin{array}{l}\text { Dery \& } \\
\text { Szivak }\end{array}$ \\
\hline $\begin{array}{l}\text { Chaetopteryx } \\
\text { rugulosa noricum }\end{array}$ & $\begin{array}{l}\text { AUT, Saualpe, springs of the } \\
\text { Klippitzbach stream near } \\
\text { Klippitztörl 21.11.2009 }\end{array}$ & CRNDI0201 & JF891188 & $\begin{array}{l}\text { Dery \& } \\
\text { Szivak }\end{array}$ \\
\hline $\begin{array}{l}\text { Chaetopteryx } \\
\text { rugulosa noricum }\end{array}$ & $\begin{array}{l}\text { AUT, Saualpe, springs of the } \\
\text { Klippitzbach stream near } \\
\text { Klippitztörl 21.11.2009 }\end{array}$ & CRNDI0202 & JF891189 & $\begin{array}{l}\text { Dery \& } \\
\text { Szivak }\end{array}$ \\
\hline
\end{tabular}




\begin{tabular}{|c|c|c|c|c|}
\hline Species name & Locality & Specimen ID & $\begin{array}{c}\begin{array}{c}\text { Accession } \\
\text { number }\end{array} \\
\end{array}$ & \begin{tabular}{|c} 
Collectors/ \\
Source
\end{tabular} \\
\hline $\begin{array}{l}\text { Chaetopteryx } \\
\text { rugulosa noricum }\end{array}$ & $\begin{array}{l}\text { AUT, Saualpe, springs of the } \\
\text { Klippitzbach stream near } \\
\text { Klippitztörl 21.11.2009 }\end{array}$ & CRNDI0203 & JF891219 & $\begin{array}{l}\text { Dery \& } \\
\text { Szivak }\end{array}$ \\
\hline $\begin{array}{l}\text { Chaetopteryx } \\
\text { rugulosa noricum }\end{array}$ & $\begin{array}{l}\text { AUT, Saualpe, springs of the } \\
\text { Klippitzbach stream near } \\
\text { Klippitztörl 21.11.2009 }\end{array}$ & CRNDI0204 & JF891220 & $\begin{array}{l}\text { Dery \& } \\
\text { Szivak }\end{array}$ \\
\hline $\begin{array}{l}\text { Chaetopteryx } \\
\text { rugulosa noricum }\end{array}$ & $\begin{array}{l}\text { AUT, Saulape, spring of the } \\
\text { Löllingbach stream near Stranach, } \\
21.11 .2009\end{array}$ & CRNDI0301 & JF891190 & $\begin{array}{l}\text { Dery \& } \\
\text { Szivak }\end{array}$ \\
\hline $\begin{array}{l}\text { Chaetopteryx } \\
\text { rugulosa noricum }\end{array}$ & $\begin{array}{l}\text { AUT, Saulape, spring of the } \\
\text { Löllingbach stream near Stranach, } \\
21.11 .2009\end{array}$ & CRNDI0302 & JF891191 & $\begin{array}{l}\text { Dery \& } \\
\text { Szivak }\end{array}$ \\
\hline $\begin{array}{l}\text { Chaetopteryx } \\
\text { rugulosa noricum }\end{array}$ & $\begin{array}{l}\text { AUT, Saulape, spring of the } \\
\text { Löllingbach stream near Stranach, } \\
21.11 .2009\end{array}$ & CRNDI0303 & JF891217 & $\begin{array}{l}\text { Dery \& } \\
\text { Szivak }\end{array}$ \\
\hline $\begin{array}{l}\text { Chaetopteryx } \\
\text { rugulosa noricum }\end{array}$ & $\begin{array}{l}\text { AUT, Saulape, spring of the } \\
\text { Löllingbach stream near Stranach, } \\
21.11 .2009\end{array}$ & CRNDI0304 & JF891218 & $\begin{array}{l}\text { Dery \& } \\
\text { Szivak }\end{array}$ \\
\hline $\begin{array}{l}\text { Chaetopteryx } \\
\text { rugulosa rugulosa }\end{array}$ & \begin{tabular}{|l|} 
HUN, Köszegi Mts., Hörmann \\
spring near Velem, 18.11.2009
\end{tabular} & CRRDJ0101 & & Szivak \\
\hline $\begin{array}{l}\text { Chaetopteryx } \\
\text { rugulosa rugulosa }\end{array}$ & $\begin{array}{l}\text { HUN, Köszegi Mts., Hörmann } \\
\text { spring near Velem, 18.11.2009 }\end{array}$ & CRRDJ0102 & & Szivak \\
\hline $\begin{array}{l}\text { Chaetopteryx } \\
\text { rugulosa rugulosa }\end{array}$ & $\begin{array}{l}\text { AUT, Mitterneuwald, Hermann } \\
\text { spring, 19.11.2009 }\end{array}$ & CRRDJ0201 & JF891184 & $\begin{array}{l}\text { Dery \& } \\
\text { Szivak }\end{array}$ \\
\hline $\begin{array}{l}\text { Chaetopteryx } \\
\text { rugulosa rugulosa }\end{array}$ & $\begin{array}{l}\text { AUT, Sommeralm, Mixnitzbach } \\
\text { stream, 20.11.2009 }\end{array}$ & CRRDJ0301 & & $\begin{array}{l}\text { Dery \& } \\
\text { Szivak }\end{array}$ \\
\hline $\begin{array}{l}\text { Chaetopteryx } \\
\text { rugulosa rugulosa }\end{array}$ & $\begin{array}{l}\text { AUT, Sommeralm, Mixnitzbach } \\
\text { stream, 20.11.2009 }\end{array}$ & CRRDJ0302 & JF891214 & $\begin{array}{l}\text { Dery \& } \\
\text { Szivak }\end{array}$ \\
\hline $\begin{array}{l}\text { Chaetopteryx } \\
\text { rugulosa rugulosa }\end{array}$ & $\begin{array}{l}\text { AUT, Hochegg bei } \\
\text { Grimmenstein, spring and its } \\
\text { outlet, 19.11.2009 }\end{array}$ & CRRDJ0401 & JF891205 & $\begin{array}{l}\text { Dery \& } \\
\text { Szivak }\end{array}$ \\
\hline $\begin{array}{l}\text { Chaetopteryx } \\
\text { rugulosa rugulosa }\end{array}$ & $\begin{array}{l}\text { AUT, Hochegg bei } \\
\text { Grimmenstein, spring and its } \\
\text { outlet, 19.11.2009 }\end{array}$ & CRRDJ0402 & JF891206 & $\begin{array}{l}\text { Dery \& } \\
\text { Szivak }\end{array}$ \\
\hline $\begin{array}{l}\text { Chaetopteryx } \\
\text { rugulosa rugulosa }\end{array}$ & $\begin{array}{l}\text { AUT, Hochegg bei } \\
\text { Grimmenstein, spring and its } \\
\text { outlet, 19.11.2009 }\end{array}$ & CRRDJ0403 & JF891207 & $\begin{array}{l}\text { Dery \& } \\
\text { Szivak }\end{array}$ \\
\hline $\begin{array}{l}\text { Chaetopteryx } \\
\text { rugulosa rugulosa }\end{array}$ & $\begin{array}{l}\text { AUT, Ausserneuwald, spring, } \\
\text { 19.11.2009 }\end{array}$ & CRRDJ0501 & JF891208 & $\begin{array}{l}\text { Dery \& } \\
\text { Szivak }\end{array}$ \\
\hline $\begin{array}{l}\text { Chaetopteryx } \\
\text { rugulosa rugulosa }\end{array}$ & $\begin{array}{l}\text { AUT, Ausserneuwald, spring, } \\
\text { 19.11.2009 }\end{array}$ & CRRDJ0502 & JF891209 & $\begin{array}{l}\text { Dery \& } \\
\text { Szivak }\end{array}$ \\
\hline $\begin{array}{l}\text { Chaetopteryx } \\
\text { rugulosa rugulosa }\end{array}$ & $\begin{array}{l}\text { AUT, Plenzengreith, upper } \\
\text { reach of stream Schöcklbach, } \\
20.11 .2009\end{array}$ & CRRDJ0601 & JF891230 & $\begin{array}{l}\text { Dery \& } \\
\text { Szivak }\end{array}$ \\
\hline $\begin{array}{l}\text { Chaetopteryx } \\
\text { rugulosa rugulosa }\end{array}$ & $\begin{array}{l}\text { AUT, Plenzengreith, upper } \\
\text { reach of stream Schöcklbach, } \\
20.11 .2009\end{array}$ & CRRDJ0602 & JF891231 & $\begin{array}{l}\text { Dery \& } \\
\text { Szivak }\end{array}$ \\
\hline $\begin{array}{l}\text { Chaetopteryx } \\
\text { rugulosa rugulosa }\end{array}$ & $\begin{array}{l}\text { AUT, Plenzengreith, upper } \\
\text { reach of stream Schöcklbach, } \\
20.11 .2009\end{array}$ & CRRDJ0603 & JF891232 & $\begin{array}{l}\text { Dery \& } \\
\text { Szivak }\end{array}$ \\
\hline $\begin{array}{l}\text { Chaetopteryx } \\
\text { rugulosa rugulosa }\end{array}$ & $\begin{array}{l}\text { SLO, Pohorje Mts., Osankarica } \\
\text { (Lukanja), 10.11.2008 }\end{array}$ & CRRDG0101 & JF891186 & Popijač \\
\hline $\begin{array}{l}\text { Chaetopteryx } \\
\text { rugulosa rugulosa }\end{array}$ & $\begin{array}{l}\text { SLO, Pohorje Mts., Osankarica } \\
\text { (Lukanja), 10.11.2008 }\end{array}$ & CRRDG0102 & JF891215 & Popijač \\
\hline
\end{tabular}




\begin{tabular}{|c|c|c|c|c|}
\hline Species name & Locality & Specimen ID & $\begin{array}{c}\text { Accession } \\
\text { number }\end{array}$ & $\begin{array}{l}\text { Collectors/ } \\
\text { Source }\end{array}$ \\
\hline $\begin{array}{l}\text { Chaetopteryx } \\
\text { rugulosa rugulosa }\end{array}$ & $\begin{array}{l}\text { SLO, Pohorje Mts., Osankarica } \\
\text { (Lukanja), 10.11.2008 }\end{array}$ & CRRDG0103 & JF891216 & Popijač \\
\hline $\begin{array}{l}\text { Chaetopteryx } \\
\text { rugulosa rugulosa }\end{array}$ & $\begin{array}{l}\text { CRO, Medvednica Mts., Mrzlak } \\
\text { spring near Sljeme, 18.11.2006 }\end{array}$ & CRREE0101 & JF891185 & Popijač \\
\hline $\begin{array}{l}\text { Chaetopteryx } \\
\text { rugulosa rugulosa }\end{array}$ & \begin{tabular}{|l|} 
CRO, Medvednica Mts., Mrzlak \\
spring near Sljeme, 18.11.2006
\end{tabular} & CRREE0102 & JF891213 & Popijač \\
\hline $\begin{array}{l}\text { Chaetopteryx } \\
\text { rugulosa rugulosa }\end{array}$ & $\begin{array}{l}\text { CRO, Medvednica Mts., Kraljičin } \\
\text { Zdenac spring, Kraljičin Zdenac, } \\
\text { 19.11.2009 }\end{array}$ & CRREE0201 & JF891210 & $\begin{array}{l}\text { Kučinić \& } \\
\text { Vučković }\end{array}$ \\
\hline $\begin{array}{l}\text { Chaetopteryx } \\
\text { rugulosa rugulosa }\end{array}$ & $\begin{array}{l}\text { CRO, Medvednica Mts., Bliznec } \\
\text { stream, Podsljeme (Pilana), } \\
\text { 09.12.2009 }\end{array}$ & CRREE0301 & JF891211 & $\begin{array}{l}\text { Kučinić \& } \\
\text { Vučković }\end{array}$ \\
\hline $\begin{array}{l}\text { Chaetopteryx } \\
\text { rugulosa rugulosa }\end{array}$ & \begin{tabular}{|l|} 
CRO, Žumberak Mts., Slapnica \\
stream, Ribička kuća, 28.10.2009
\end{tabular} & CRREF0101 & JF891212 & $\begin{array}{l}\text { Kučinić \& } \\
\text { Vučković }\end{array}$ \\
\hline $\begin{array}{l}\text { Chaetopteryx } \\
\text { schmidi }\end{array}$ & $\begin{array}{l}\text { ROU, spring brook in Cerna } \\
\text { valley near Tatu, 13.11.2010 }\end{array}$ & CRSJF0101 & HE858259 & $\begin{array}{l}\text { Ecsedi \& } \\
\text { Szivak }\end{array}$ \\
\hline $\begin{array}{l}\text { Chaetopteryx } \\
\text { schmidi }\end{array}$ & $\begin{array}{l}\text { ROU, spring brook in Cerna } \\
\text { valley near Tatu, 13.11.2010 }\end{array}$ & CRSJF0102 & HE858260 & \begin{tabular}{|l|} 
Ecsedi \& \\
Szivak
\end{tabular} \\
\hline $\begin{array}{l}\text { Chaetopteryx } \\
\text { schmidi }\end{array}$ & $\begin{array}{l}\text { ROU, spring brook in Cerna } \\
\text { valley near Tatu, 13.11.2010 }\end{array}$ & CRSJF0103 & HE858261 & $\begin{array}{l}\text { Ecsedi \& } \\
\text { Szivak }\end{array}$ \\
\hline $\begin{array}{l}\text { Chaetopteryx } \\
\text { schmidi }\end{array}$ & $\begin{array}{l}\text { SRB, Derdap Mts., stream valley } \\
\text { N of Golubinje, } 13.10 .2006\end{array}$ & CRSGE0101 & JF891182 & $\begin{array}{l}\text { Danyi, } \\
\text { Kontschan \& } \\
\text { Muranyi }\end{array}$ \\
\hline $\begin{array}{l}\text { Chaetopteryx } \\
\text { schmidi }\end{array}$ & $\begin{array}{l}\text { SRB, Derdap Mts., stream valley } \\
\text { N of Golubinje, } 13.10 .2006\end{array}$ & CRSGE0102 & JF891201 & $\begin{array}{l}\text { Danyi, } \\
\text { Kontschan \& } \\
\text { Muranyi }\end{array}$ \\
\hline $\begin{array}{l}\text { Chaetopteryx } \\
\text { schmidi }\end{array}$ & $\begin{array}{l}\text { SRB, Derdap Mts.,Grgeci spring, } \\
\text { Donji Milankovac, } 13.10 .2006\end{array}$ & CRSGE0201 & JF891183 & \begin{tabular}{|l|} 
Danyi, \\
Kontschan \& \\
Muranyi \\
\end{tabular} \\
\hline $\begin{array}{l}\text { Chaetopteryx } \\
\text { schmidi }\end{array}$ & \begin{tabular}{|l|} 
SRB, Derdap Mts., Grgeci spring, \\
Donji Milankovac, 13.10 .2006
\end{tabular} & CRSGE0203 & JF891202 & $\begin{array}{l}\text { Danyi, } \\
\text { Kontschan \& } \\
\text { Muranyi } \\
\end{array}$ \\
\hline $\begin{array}{l}\text { Chaetopteryx bucari } \\
\text { sp. n. }\end{array}$ & $\begin{array}{l}\text { CRO, Kriz spring near Petrinja, } \\
08.12 .2009\end{array}$ & CxxEC0101 & JF891192 & $\begin{array}{l}\text { Kučinić, } \\
\text { Delić \& } \\
\text { Bučar } \\
\end{array}$ \\
\hline $\begin{array}{l}\text { Chaetopteryx bucari } \\
\text { sp. n. }\end{array}$ & $\begin{array}{l}\text { CRO, Kriz spring near Petrinja, } \\
07.11 .2009\end{array}$ & CxxEC0102 & JF891222 & $\begin{array}{l}\text { Kučinić, } \\
\text { Delić \& } \\
\text { Bučar }\end{array}$ \\
\hline $\begin{array}{l}\text { Chaetopteryx bucari } \\
\text { sp. n. }\end{array}$ & $\begin{array}{l}\text { CRO, Kriz spring near Petrinja, } \\
07.11 .2009\end{array}$ & CxxEC0103 & JF891223 & $\begin{array}{l}\text { Kučinić, } \\
\text { Delić \& } \\
\text { Bučar }\end{array}$ \\
\hline $\begin{array}{l}\text { Chaetopteryx bucari } \\
\text { sp. n. }\end{array}$ & $\begin{array}{l}\text { CRO, Kriz spring near Petrinja, } \\
04.11 .2009\end{array}$ & CxxEC0104 & JF891224 & Bučar \\
\hline $\begin{array}{l}\text { Chaetopteryx bucari } \\
\text { sp. n. }\end{array}$ & $\begin{array}{l}\text { CRO, Kriz spring near Petrinja, } \\
\text { 08.12.2009 }\end{array}$ & CxxEC0105 & JF891225 & $\begin{array}{l}\text { Kučinić, } \\
\text { Delić, Bučar } \\
\text { \& Vučković }\end{array}$ \\
\hline $\begin{array}{l}\text { Chaetopteryx bucari } \\
\text { sp. n. }\end{array}$ & $\begin{array}{l}\text { CRO, Hrvatski Cuntic, Marića } \\
\text { točak spring, 22.11.2009 }\end{array}$ & CxxEC0201 & JF891193 & \begin{tabular}{|l|} 
Kučinić, \\
Delić \& \\
Bučar \\
\end{tabular} \\
\hline $\begin{array}{l}\text { Chaetopteryx bucari } \\
\text { sp. n. }\end{array}$ & $\begin{array}{l}\text { CRO, Hrvatski Cuntic, Marića } \\
\text { točak spring, 21.11.2009 }\end{array}$ & CxxEC0202 & JF891221 & $\begin{array}{l}\text { Kučinić, } \\
\text { Delić \& } \\
\text { Bučar }\end{array}$ \\
\hline $\begin{array}{l}\text { Chaetopteryx bucari } \\
\text { sp. n. }\end{array}$ & \begin{tabular}{|l|} 
CRO, Hrvatska Kostajnica, \\
Varoški bunar spring, 06.12.2009
\end{tabular} & CxxEC0301 & & $\begin{array}{l}\text { Kučinić, } \\
\text { Delić \& } \\
\text { Bučar }\end{array}$ \\
\hline
\end{tabular}




\begin{tabular}{|c|c|c|c|c|}
\hline Species name & Locality & Specimen ID & $\begin{array}{c}\text { Accession } \\
\text { number }\end{array}$ & $\begin{array}{c}\text { Collectors/ } \\
\text { Source }\end{array}$ \\
\hline $\begin{array}{l}\text { Chaetopteryx bucari } \\
\text { sp. n. }\end{array}$ & $\begin{array}{l}\text { CRO, Šuplji Kamen, Slabinja } \\
\text { spring, 29.11.2009 }\end{array}$ & CxxEC0401 & JF891194 & \begin{tabular}{|l|} 
Kučinić, \\
Delić \& \\
Bučar
\end{tabular} \\
\hline $\begin{array}{l}\text { Chaetopteryx bucari } \\
\text { sp. n. }\end{array}$ & $\begin{array}{l}\text { CRO, Banovina region, Pecki } \\
\text { spring, 15.12.2009 }\end{array}$ & CxxEC0501 & JF891195 & $\begin{array}{l}\text { Kučinić, } \\
\text { Delić \& } \\
\text { Bučar }\end{array}$ \\
\hline $\begin{array}{l}\text { Chaetopteryx bucari } \\
\text { sp. n. }\end{array}$ & $\begin{array}{l}\text { CRO, Banovina region, Pecki } \\
\text { spring, 21.11.2009 }\end{array}$ & CxxEC0502 & JF891228 & $\begin{array}{l}\text { Kučinić, } \\
\text { Delić \& } \\
\text { Bučar } \\
\end{array}$ \\
\hline $\begin{array}{l}\text { Chaetopteryx bucari } \\
\text { sp. n. }\end{array}$ & $\begin{array}{l}\text { CRO, Banovina region, Pecki } \\
\text { spring, 21.11.2009 }\end{array}$ & CxxEC0503 & JF891229 & $\begin{array}{l}\text { Kučinić, } \\
\text { Delić \& } \\
\text { Bučar } \\
\end{array}$ \\
\hline $\begin{array}{l}\text { Chaetopteryx bucari } \\
\text { sp. n. }\end{array}$ & $\begin{array}{l}\text { CRO, Banovina region, Gora } \\
\text { spring, 10.12.2009 }\end{array}$ & CxxEC0601 & JF891226 & Bučar \\
\hline $\begin{array}{l}\text { Chaetopteryx bucari } \\
\text { sp. n. }\end{array}$ & $\begin{array}{l}\text { CRO, Mečenčani, Pašino vrelo, } \\
\text { 29.11.2009 }\end{array}$ & CxxEC0701 & JF891227 & $\begin{array}{l}\text { Kučinić, } \\
\text { Delić \& } \\
\text { Bučar }\end{array}$ \\
\hline $\begin{array}{l}\text { Chaetopterygopsis } \\
\text { maclachlani }\end{array}$ & $\begin{array}{l}\text { AUT, Lower Austria, } \\
\text { Rohrwiesteich, 20.10.2004 }\end{array}$ & 08HMCAD-331* & HMTRI331-09* & Malicky \\
\hline Chaetopteryx fusca & $\begin{array}{l}\text { AUT, Lower Austria, } \\
\text { Rohrwiesteich, 20.10.2004 }\end{array}$ & 08HMCAD-333* & HMTRI333-09* & Malicky \\
\hline Chaetopteryx gessneri & $\begin{array}{l}\text { ITA, Umbria, Perugia, Fium Nera } \\
\text { above Visso,11.12.2005 }\end{array}$ & 07HMCAD-0177* & HMCAD177-08* & Malicky \\
\hline Chaetopteryx moretti & $\begin{array}{l}\text { ITA, Belluno, Val Canzoi, Veneto, } \\
31.10 .2003\end{array}$ & HM09Cm7* & HMTRI421-09* & Malicky \\
\hline Chaetopteryx villosa & \begin{tabular}{|l|} 
AUT, Lower Austria, \\
Sarleinsbach, 27.06.2005 \\
\end{tabular} & 07HMCAD-0134* & HMCAD134-08* & Malicky \\
\hline Drusus alpinus & $\begin{array}{l}\text { IT, Valprato Soana, Ronchietto, } \\
10.07 .2004\end{array}$ & HM09Dalp8* & HMTRI456-09* & Delmaistro \\
\hline Drusus discolor & $\begin{array}{l}\text { SK, Lower Tatra, Stream above } \\
\text { Partizanska L'upča, 09.06.2008 }\end{array}$ & ESCAD909-17* & KKCAD497-09* & Bonada \\
\hline Drusus rectus & $\begin{array}{l}\text { ES, Camprodon/Setcases Alta Val } \\
\text { de Ter, 27.07.2004 }\end{array}$ & HM09Drec8* & HMTRI423-09* & Aistleitner \\
\hline Metanoea rhaetica & $\begin{array}{l}\text { AUT, Carinthia, Valentinbach, } \\
\text { Plockenstrasse, 08.07.2007 }\end{array}$ & 08HMCAD-020* & HMTRI020-08* & Malicky \\
\hline $\begin{array}{l}\text { Limnephilus } \\
\text { centralis }\end{array}$ & NORWAY & NHRS:FI9 & FN601020 & \begin{tabular}{|l} 
Malm \& \\
Johanson \\
2011
\end{tabular} \\
\hline
\end{tabular}

versible model (Rodríguez et al. 1990) with a significant proportion of invariant sites $(\mathrm{I}=0.607)$ and with gamma-distributed rate heterogeneity $(\alpha=1.049)(\mathrm{GTR}+\mathrm{I}+\mathrm{G})$. We conducted Bayesian tree construction with 6 chains, 2 independent runs and 8 million generations. Trees were sampled every 1000th generation. The first 9000 generations were discarded as burn-in. We plotted the log-likelihood scores of sample points against generation time using Tracer 1.5 (Rambaut and Drummond 2009) to ensure that stationary was achieved after the first 9000 generations by checking whether the $\log$-likelihood values of the sample points reached a stable equilibrium plateau. We used the remaining trees with average branch lengths to create a $50 \%$ majority-rule consensus tree with the sumt option of MrBayes. Posterior probabilities (pp) were obtained for each clade, whereby $\mathrm{pp} \geq 0.95$ indicated significant support for clades. Finally, we also calculated the uncorrected pairwise distances between individuals based on mtCOI sequences using MEGA 5.1 (Tamura et al. 2011). 


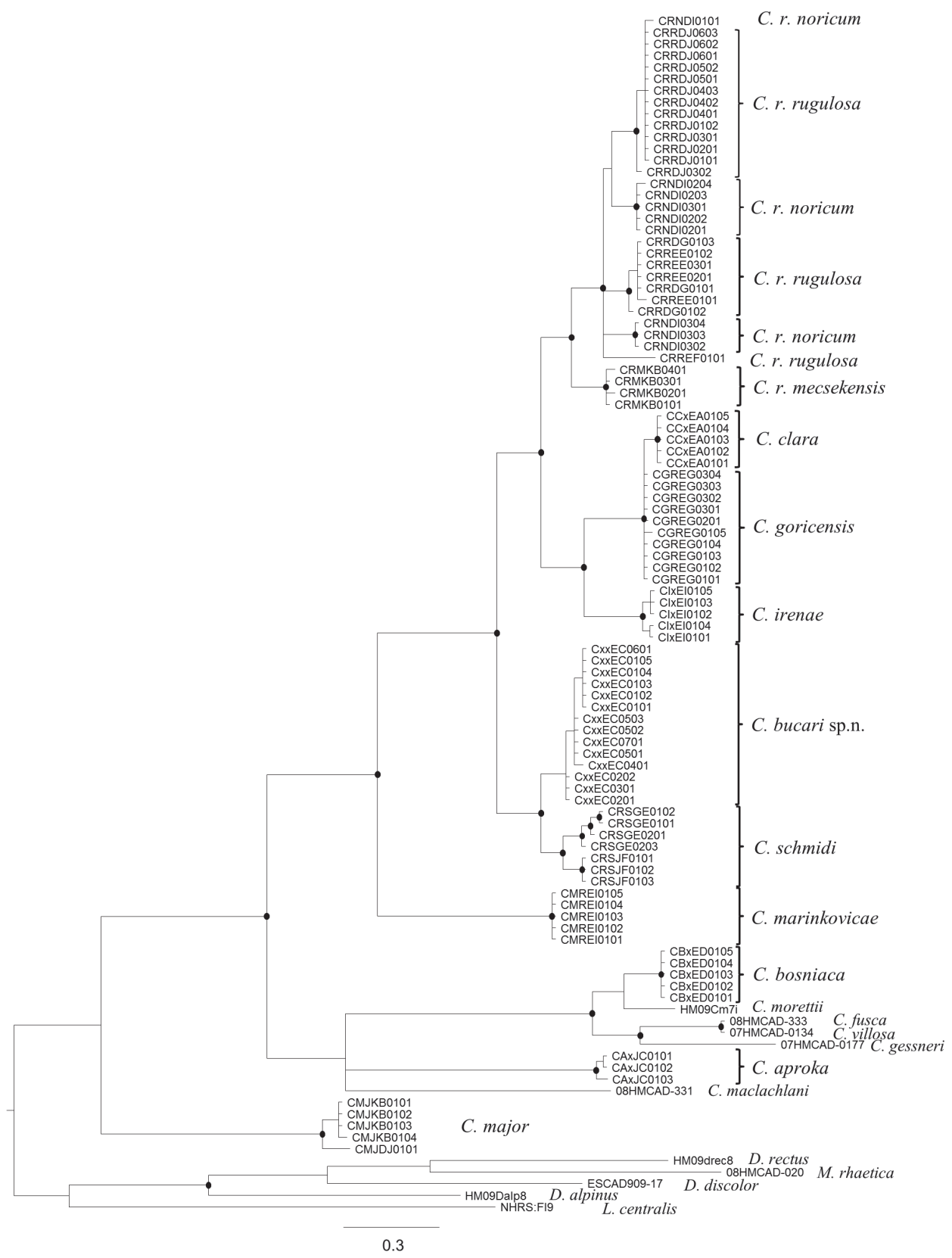

Figure 2. Bayesian tree for members of the Chaetopteryx rugulosa species group based on mitochondrial COI sequence. Black circles on nodes mark Bayesian posterior probabilities pp $>0.95$.

Microphotography and measuring. Microphotographic images of genitalia and forewing measurements were taken using a Leica Wild MZ8 stereomicroscope and Olympus SP-500 UZ digital camera. The photographs were processed with 
the Olympus Quick Photo Camera 2.2. software package. Geographic coordinates and altitudes of sampling localities were recorded with a Garmin 'Oregon 450' GPS device.

\section{Results}

Phylogenetic analyses. In the Bayesian phylogenetic tree based on mtCOI sequences the C. rugulosa group species clustered into 4 strongly supported clades (Figure 2). Chaetopteryx marinkovicae was basal within the species group. The remaining species fell into 3 clades: a basal clade with C. r. schmidi, C. bucari sp. n., and 2 derived sister clades comprising C. clara, C. goricensis, C. irenae, and C.r.rugulosa, C. r. noricum, C. r. mecsekensis. Chaetopteryx bucari sp. $\mathrm{n}$. is sister to the highly supported $C$. r. schmidi. The mean value of the uncorrected pairwise distance (p distance) was $2.02 \%$ between them (Table 3 ). The p distance did not reach $1 \%$ within the 2 clades (C. bucari sp. n.: 0.17\%; C. r. schmidi: 0.75\%). The relationship of the nominal species of the group C. r. rugulosa and C. r. noricum was not resolved, as the 4 subclades formed a polytomy. In the phylogenetic tree C. r. schmidi was clearly separated from the clade containing the subspecies of $C$. rugulosa (Figure 2). The mean values of $\mathrm{p}$ distance between the 3 subspecies of $C$. rugulosa ranged between 1.61-3.02\%, while the mean values between the C. $r$. schmidi and the other subspecies of $C$. rugulosa were distinctly higher (4.66-5.85\%) (Table 3).

\section{Chaetopteryx bucari Kučinić, Szivák \& Delić, sp. n.} http://zoobank.org/E775EC69-0E8A-4AF0-A027-F290BB31E76E http://species-id.net/wiki/Chaetopteryx_bucari

Figures 3-16

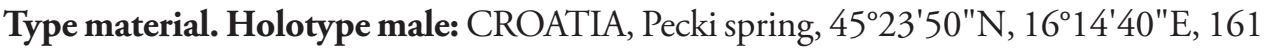
$m$ a.s.l., 15 December 2009, leg. Bučar, Delić, Kučinić, dry specimen, DNA Barcode ID: HGCAD046-10, deposited in the Croatian Natural History Museum in Zagreb.

Paratype: CROATIA, $\delta$ and $q(\mathrm{n}=49): 1$ female, Pecki spring, 21 November 2009, leg. Bučar, Delić, Kučinić, dry specimen, DNA Barcode ID: HGCAD087-10; 14 males, Pecki spring, 31 October 2011; 9 females, Pecki spring, 31 October 2010; 20 females, Pecki spring, 30 November 2011; 2 males and 2 females, Hrvatski Čuntić stream, $45^{\circ} 21^{\prime} 28^{\prime \prime} \mathrm{N}, 16^{\circ} 17^{\prime} 04^{\prime \prime} \mathrm{E}, 159 \mathrm{~m}$ a.s.l., 22 October 2010; 1 male, Marića

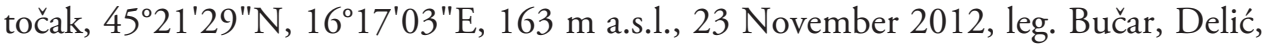
Kučinić (all specimens in alcohol).

Diagnosis. Male of C. bucari is most similar to C. r. mecsekensis and C. r. schmidi but differs in the following features: 1 . In lateral view the inferior appendages in $C$. bucari are always with a pointed apex on the dorsal side, not rounded as in C. r. mecsekensis; 2 . Bristles in C. bucari are set more distally from the membranous part of the aedeagus than in C.r.mecsekensis and C.r. schmidi and never reach (touch) the lateral 
Table 3. Estimates of evolutionary divergence over sequence pairs within and between phylogenetically defined species and subspecies based on mtCOI sequence data. Distance matrix is shown with the mean $\pm \mathrm{SD}$ values of the intraspecific and interspecific pairwise genetic distances for the all Chaetopterygini species included in the analysis. Abbrev.: CRR - Chaetopteryx rugulosa rugulosa, CRN - C. r. noricum, CRM - C. r. mecsekensis, CCX - C. clara, CGR - C. goricensis, CIX - C. irenae, CBU - C. bucari sp.n., CRS - C. schmidi, CMR - C. marinkovicae, $\mathrm{CBO}-$ C. bosniaca, CMO - C. morettii, CFU - C. fusca, CVI - C. villosa, CGE - C. gessneri, CAX - C. aproka, CMA - Chaetopterygopsis maclachlani, CMJ Chaetopteryx major.

\begin{tabular}{|c|c|c|c|c|c|c|c|c|c|c|c|}
\hline & CRR & CRN & CRM & \multicolumn{2}{|c|}{ CCX } & \multicolumn{2}{|l|}{ CGR } & CIX & CBU & CRS & CMR \\
\hline CRR & \begin{tabular}{|l|l}
$1.05 \pm 0.97$ & 1 \\
\end{tabular} & \begin{tabular}{|l|l|}
$1.61 \pm 0.49$ & 3. \\
\end{tabular} & \multirow{2}{*}{\begin{tabular}{|l|}
$3.02 \pm 0.17$ \\
$2.83 \pm 0.31$ \\
\end{tabular}} & \multicolumn{2}{|c|}{$4.87 \pm 0.24$} & \multicolumn{2}{|c|}{$4.55 \pm 0.24$} & $4.79 \pm 0.27$ & \begin{tabular}{l|l}
0.27 & $4.63 \pm 0.30$ \\
\end{tabular} & $5.44 \pm 0.39$ & $9.24 \pm 0.46$ \\
\hline CRN & & \begin{tabular}{|l|l|}
$1.20 \pm 0.89$ & .
\end{tabular} & & \multicolumn{2}{|c|}{$5.06 \pm 0.23$} & \multicolumn{2}{|c|}{$4.74 \pm 0.24$} & $5.26 \pm 0.20$ & \begin{tabular}{l|l|}
.20 & $5.17 \pm 0.18$ \\
\end{tabular} & $5.85 \pm 0.11$ & $9.45 \pm 0.27$ \\
\hline CRM & & & $0.17 \pm 0.11$ & \multicolumn{2}{|c|}{$4.79 \pm 0.09$} & \multicolumn{2}{|c|}{$4.47 \pm 0.10$} & $4.69 \pm 0.12$ & \begin{tabular}{l|l}
0.12 & $4.38 \pm 0.11$
\end{tabular} & $4.66 \pm 0.13$ & $8.87 \pm 0.07$ \\
\hline $\mathrm{CCX}$ & & & & \multicolumn{2}{|c|}{$0.00 \pm 0.00$} & \multicolumn{2}{|c|}{$0.37 \pm 0.07$} & $3.80 \pm 0.08$ & \begin{tabular}{l|l|}
.08 & $5.86 \pm 0.14$ \\
\end{tabular} & $5.69 \pm 0.06$ & $9.92 \pm 0.00$ \\
\hline CGR & & & & & & \multicolumn{2}{|c|}{$0.03 \pm 0.07$} & $3.48 \pm 0.10$ & \begin{tabular}{l|l}
0.10 & $5.54 \pm 0.15$ \\
\end{tabular} & $5.37 \pm 0.08$ & $9.93 \pm 0.05$ \\
\hline CIX & & & & & & \multicolumn{2}{|c|}{\begin{tabular}{|l|} 
\\
\end{tabular}} & $0.10 \pm 0.09$ & \begin{tabular}{l|l}
.09 & $4.41 \pm 0.16$ \\
\end{tabular} & $5.11 \pm 0.18$ & $10.15 \pm 0.08$ \\
\hline $\mathrm{CBU}$ & & & & & & \multirow{2}{*}{\multicolumn{2}{|c|}{ (2) }} & \multicolumn{2}{|c|}{\begin{tabular}{|l|l|} 
& $0.17 \pm 0.13$ \\
\end{tabular}} & $2.02 \pm 0.20$ & $8.79 \pm 0.13$ \\
\hline CRS & & & & & & & & & $0.75 \pm 0.57$ & $8.72 \pm 0.06$ \\
\hline CMR & & & & & & 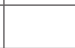 & 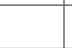 & & & & $0.00 \pm 0.00$ \\
\hline СBO & & & & & & & & & & & \\
\hline CMO & & & & & & & & & & & \\
\hline CFU & & & & & & & & & & & \\
\hline CVI & & & & & & & & & & & \\
\hline CGE & & & & & & & & & & & \\
\hline CAX & & & & & & & & & & & \\
\hline CMA & & & & & & & & & & & \\
\hline CMJ & & & & & & & & & & & \\
\hline & CBO & CMO & $\mathrm{CFU}$ & & $\overline{C V I}$ & & $\mathrm{CC}$ & $\overline{\mathrm{GE}}$ & CAX & CMA & CMJ \\
\hline CRR & $12.64 \pm 0.15$ & $12.85 \pm 0.33$ & \begin{tabular}{l|l}
3 & $12.69 \pm 0.2$ \\
\end{tabular} & & $12.69 \pm 0$ & 0.20 & 14.03 & \pm 0.20 & $12.00 \pm 0.39$ & $12.32 \pm 0.20$ & $14.17 \pm 0.16$ \\
\hline CRN & $12.85 \pm 0.08$ & $12.66 \pm 0.12$ & \begin{tabular}{|l|l|}
2 & $12.83 \pm 0.1$ \\
\end{tabular} & & $12.83 \pm 0$ & \begin{tabular}{l|l}
0.12 & 1 \\
\end{tabular} & 14.06 & \pm 0.12 & \begin{tabular}{|l|l}
$12.18 \pm 0.14$ & 1 \\
\end{tabular} & $11.98 \pm 0.24$ & $14.14 \pm 0.39$ \\
\hline CRM & $11.89 \pm 0.07$ & $11.97 \pm 0.08$ & \begin{tabular}{l|l}
8 & $11.76 \pm 0.1$ \\
\end{tabular} & & $11.76 \pm 0$ & 0.14 & 12.44 & \pm 0.14 & \begin{tabular}{|l|l}
$11.60 \pm 0.12$ & 1 \\
\end{tabular} & $11.47 \pm 0.08$ & $13.78 \pm 0.00$ \\
\hline $\mathrm{CCX}$ & $12.61 \pm 0.00$ & $12.61 \pm 0.00$ & \begin{tabular}{l|l}
0 & $12.77 \pm 0.0$
\end{tabular} & & $12.77 \pm 0$ & \begin{tabular}{l|l}
0.00 & 1
\end{tabular} & 13.61 & \pm 0.00 & $12.77 \pm 0.00$ & $2.61 \pm 0.00$ & $14.49 \pm 0.07$ \\
\hline CGR & $12.62 \pm 0.05$ & $12.62 \pm 0.05$ & \begin{tabular}{l|l}
5 & $12.79 \pm 0.0$
\end{tabular} & & $12.79 \pm 0$ & 0.05 & 13.63 & \pm 0.05 & $12.78 \pm 0.05$ & $2.62 \pm 0.05$ & $14.17 \pm 0.08$ \\
\hline CIX & $12.67 \pm 0.08$ & $13.21 \pm 0.09$ & \begin{tabular}{l|l}
9 & $12.54 \pm 0.0$
\end{tabular} & & $12.54 \pm 0$ & $=0.09$ & 13.04 & \pm 0.09 & $13.38 \pm 0.09$ & $13.55 \pm 0.09$ & $15.39 \pm 0.11$ \\
\hline CBU & $12.48 \pm 0.13$ & $12.65 \pm 0.14$ & \begin{tabular}{l|l}
4 & $12.48 \pm 0.1$ \\
\end{tabular} & & $12.48 \pm 0$ & 0.14 & 12.65 & \pm 0.14 & $11.21 \pm 0.08$ & $2.89 \pm 0.08$ & $14.00 \pm 0.16$ \\
\hline CRS & $12.24 \pm 0.06$ & \begin{tabular}{|l|l}
$53.04 \pm 0.13$ \\
\end{tabular} & \begin{tabular}{l|l}
3 & $12.05 \pm 0.2$ \\
\end{tabular} & & $12.05 \pm 0$ & 0.21 & 12.67 & \pm 0.23 & $11.56 \pm 0.22$ & $3.93 \pm 0.06$ & $14.44 \pm 0.15$ \\
\hline CMR & $11.60 \pm 0.00$ & $11.60 \pm 0.00$ & \begin{tabular}{l|l}
0 & $11.93 \pm 0.0$
\end{tabular} & & $11.93 \pm 0$ & 0.00 & 13.11 & \begin{tabular}{|l|l} 
\pm 0.00 & 1 \\
\end{tabular} & $11.76 \pm 0.00$ & $2.94 \pm 0.00$ & $12.77 \pm 0.18$ \\
\hline $\mathrm{CBO}$ & $0.00 \pm 0.00$ & $2.69 \pm 0.00$ & \begin{tabular}{l|l} 
& $4.87 \pm 0.0$
\end{tabular} & & $4.87 \pm 0$ & 0.00 & 6.05 & \begin{tabular}{l|l} 
\pm 0.00 & 1 \\
\end{tabular} & $12.61 \pm 0.00$ & $2.61 \pm 0.00$ & $15.26 \pm 0.17$ \\
\hline CMO & & $0.00 \pm 0.00$ & $5.55 \pm 0.0$ & & $5.55 \pm 0$ & 0.00 & 6.55 & \begin{tabular}{|l|l}
0.00 & 1 \\
\end{tabular} & $12.94 \pm 0.00$ & $11.76 \pm 0.00$ & $14.82 \pm 0.08$ \\
\hline CFU & & & $0.00 \pm 0.0$ & & $0.00 \pm 0$ & 0.00 & 5.88 & \begin{tabular}{|l|l} 
\pm 0.00 & 1 \\
\end{tabular} & $13.05 \pm 0.19$ & $2.94 \pm 0.00$ & $15.83 \pm 0.08$ \\
\hline CVI & & & & & $0.00 \pm 0$ & 0.00 & 5.88 & $\pm 0.00 \quad 1$ & \begin{tabular}{|l|l}
$13.05 \pm 0.19$ & 1 \\
\end{tabular} & $2.94 \pm 0.00$ & $15.83 \pm 0.08$ \\
\hline CGE & & & & & & & 0.00 & $\pm 0.00 \quad 1$ & $13.45 \pm 0.00$ & $3.44 \pm 0.00$ & $15.87 \pm 0.09$ \\
\hline CAX & & & & & & & & & \begin{tabular}{|l|l}
$0.35 \pm 0.00$ & 1 \\
\end{tabular} & $11.71 \pm 0.19$ & $13.44 \pm 0.11$ \\
\hline CMA & & & & & & & & & & $0.00 \pm 0.00$ & $13.04 \pm 0.15$ \\
\hline CMJ & & & & & & & & & & & $0.47 \pm 0.51$ \\
\hline
\end{tabular}




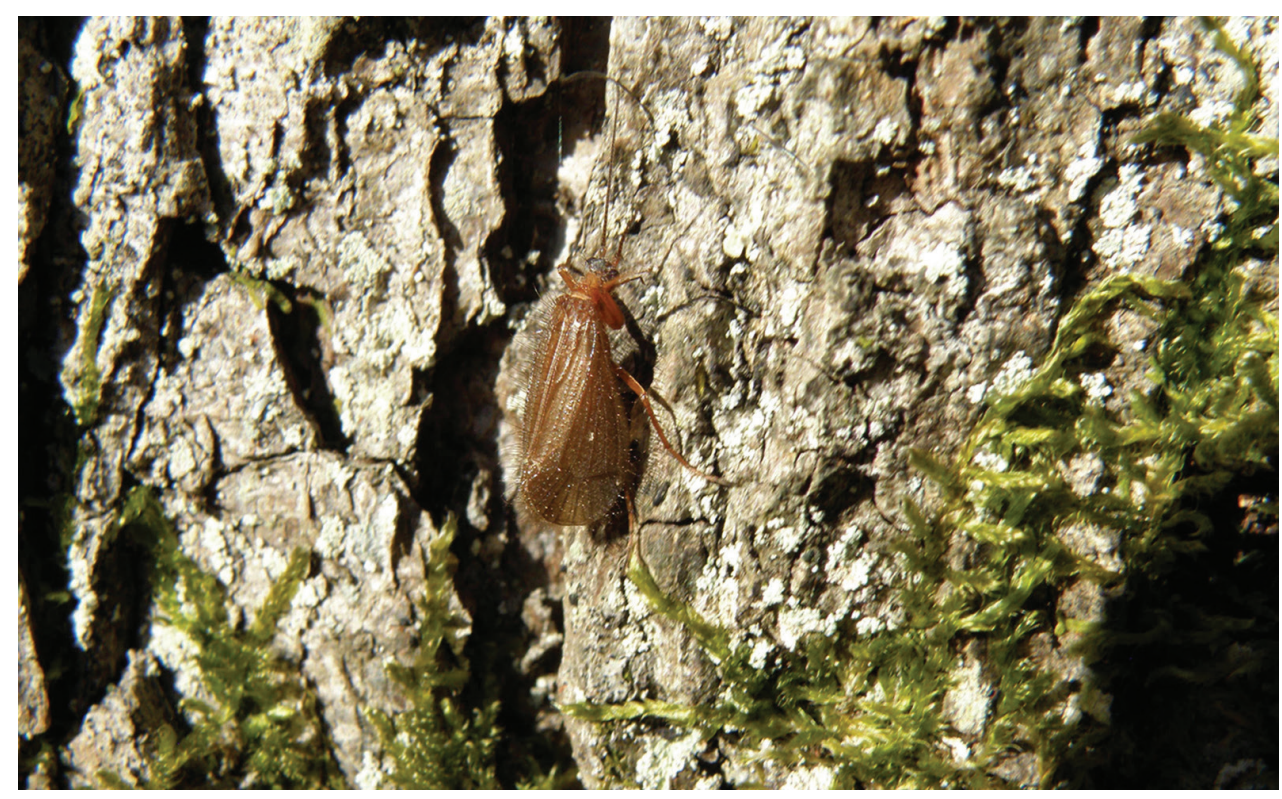

Figure 3. Chaetopteryx bucari, sp. n., adults at type locality, Pecki spring, Croatia.

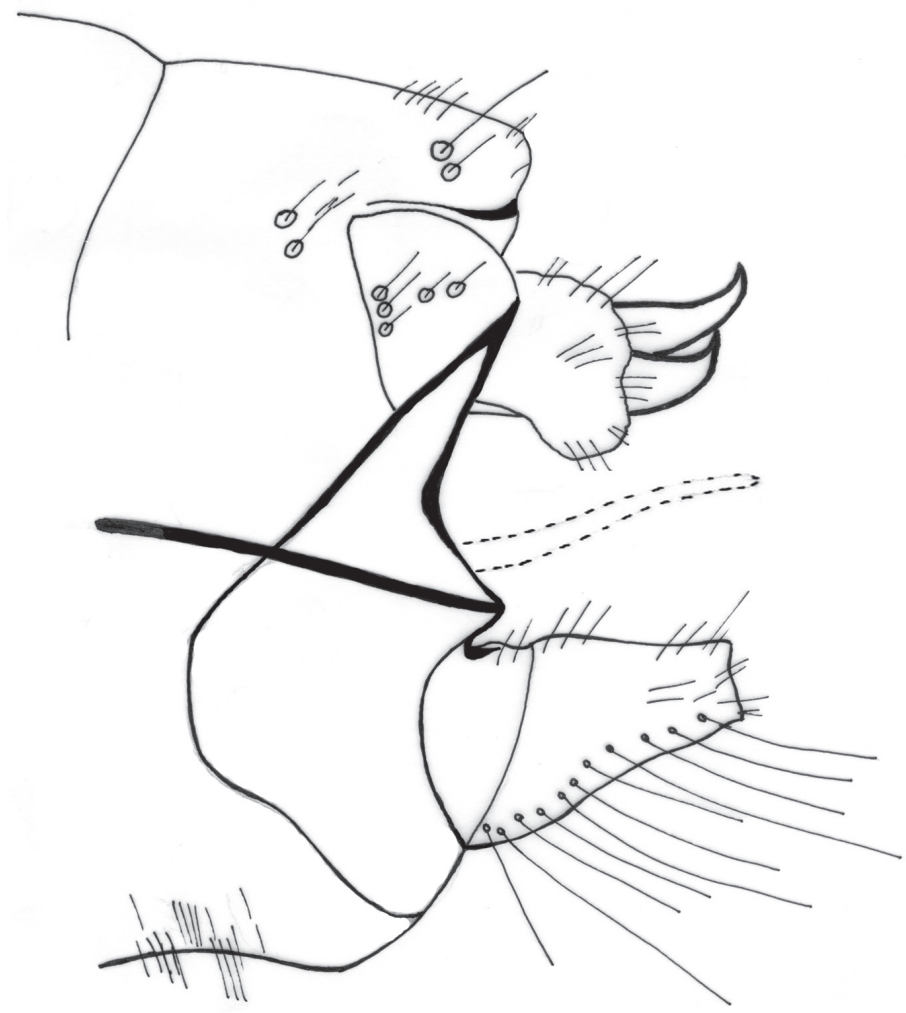

Figure 4. Chaetopteryx bucari sp. n., male genitalia, lateral view. 


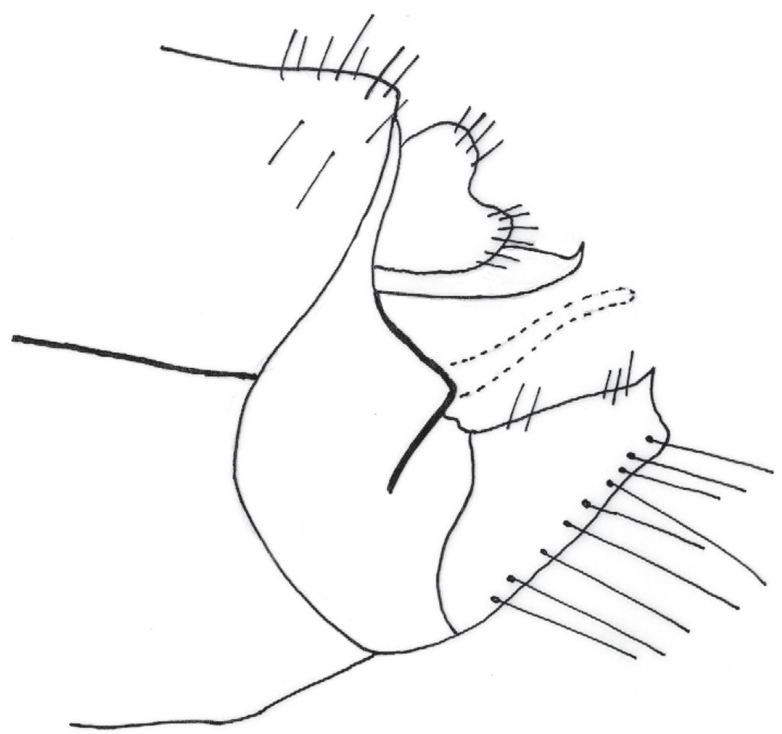

Figure 5. Chaetopteryx bucari sp. n., male genitalia, lateral view.

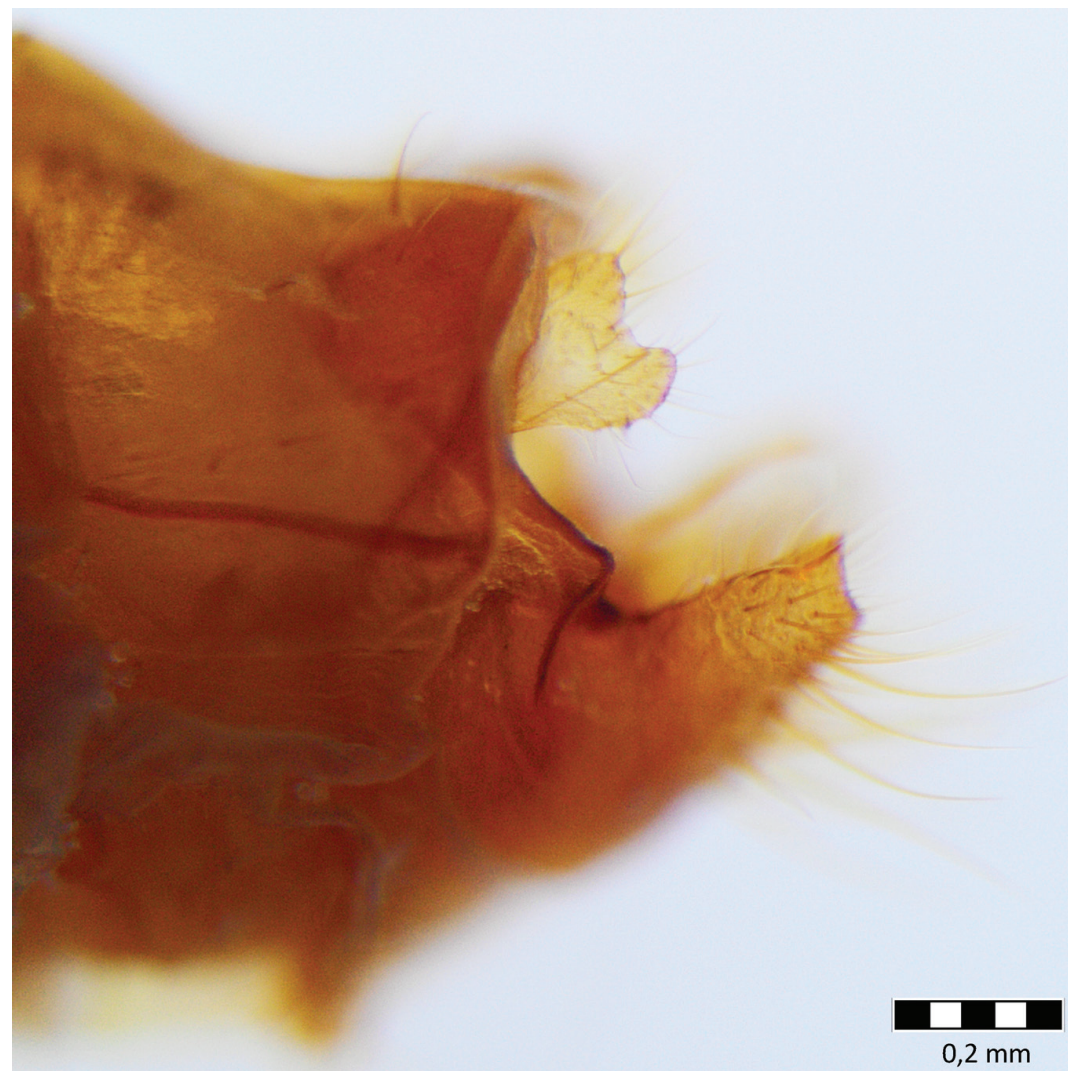

Figure 6. Chaetopteryx bucari sp. n., male genitalia, lateral view. 
membranous finger, as in C.r. mecsekensis. Female of C. bucari is clearly different from other species in the C. rugulosa group (e.g., form of the visible finger on lateral side, form of the anal tube, form of the supragenital plate of segment $\mathrm{X}$ in lateral and ventral views, form of the median lobe of the vulvar scale in ventral view). We did not find strong morphological variability among the females of the new species (except the median lobe of the vulvar scale). Females of $C$. bucari have in lateral, ventral and dorsal views very visible finger-shaped proturbances (ventral lobes of tergite IX) on the anal tube which is lacking in C.r. mecsekensis and C.r. schmidi. In lateral view the excision of the anal tube in C.r. rugulosa is more pronounced than in C. bucari. The median lobe of the vulvar scale in C.r. mecsekensis, C.r. rugulosa and C.r. schmidi is longer and more visible than in C. bucari.

Description. Wings and legs yellow to yellowish-brown; veins darker in both sexes (Figure 3). Antennae long, grey to fuscous. Scapus yellow to yellowish-brown, thorax and abdomen yellow. Spur formula male 0,3,3, female 1,3,3. Ocelli present. Forewing with round apex; length 7.7-9.9 $\mathrm{mm}$ in males, $7.2-10.1 \mathrm{~mm}$ in females.

Male genitalia (Figures 4-11). In dorsal view, spinulose zone of tergite VIII well developed with yellow setae. Segment IX ventrally broad, dorsally narrow in lateral view (Figures 4-5). Superior appendages with small yellow setae, shape of superior appendages variable (Figures 4-7b-d), usually in one of two forms (Figures 4-6). In lateral view, 1st form with posterior edge slightly rounded apically, concave at middle (Figure 5); in 2nd form, dorsal side more protuberant with round or irregular apex (Figures 4, 7b). In some specimens triangular or rectangular intermediate forms are found (Figure $7 \mathrm{c}-\mathrm{d}$ ). Inferior appendages in lateral view rectangular, anterior part broad, posterior part narrow (Figures 4-7a). Apical flap of inferior appendage developed, in lateral view with pointed apex (tip) and ventral side slightly rounded; or with apex forked, long setae present on ventral side (Figures 4-7a). Intermediate appendages (paraproctal complex) elongated in lateral view with long, connecting middle section, apical hook narrowing with upward-curving apex (Figures 4-5), basal triangular part of paraproct relatively large in caudal view (Figures 8-9). Phallic organ (phallus) a single tube consisting of phallic apodeme, phallobase, aedeagus and parameres. Aedeagus relatively long, sclerotized, in posterior part with membranous lobes, lateral lobes membranous finger-like proturbances (endophallus) (Figures 10a-d). Two relatively short parameres set very distant from posterior membranous part of aedeagus (Figures 10a-b, 10d); parameres with sclerotized, straight, stout, brown bristles (Figures 10a-b, 10d, 11a-f). Bristles vary in width and length (Figure 11a-f); lateral bristles shorter; bristles arranged in 1 fan-like row (Figure 11a-f); in specimens with more bristles, some form 2nd row; bristles vary from 5-10.

Female genitalia (Figures 12-16). Anal tube (fusion of tergites IX and X) in lateral view broad, relatively elongated with one excision and very distinct finger-shaped proturbance (lobes of tergite IX) on ventral side (Figures 12-13). Apex of proturbance rounded or slightly pointed with small yellow setae (Figures $12-15$ ). In $2 / 3$ rds of specimens examined ventral and dorsal lips of anal tube equal in length, in $1 / 3 \mathrm{rd}$ ventral lip longer. In dorsal view anal tube thickened with digitate proturbance on lateral side 

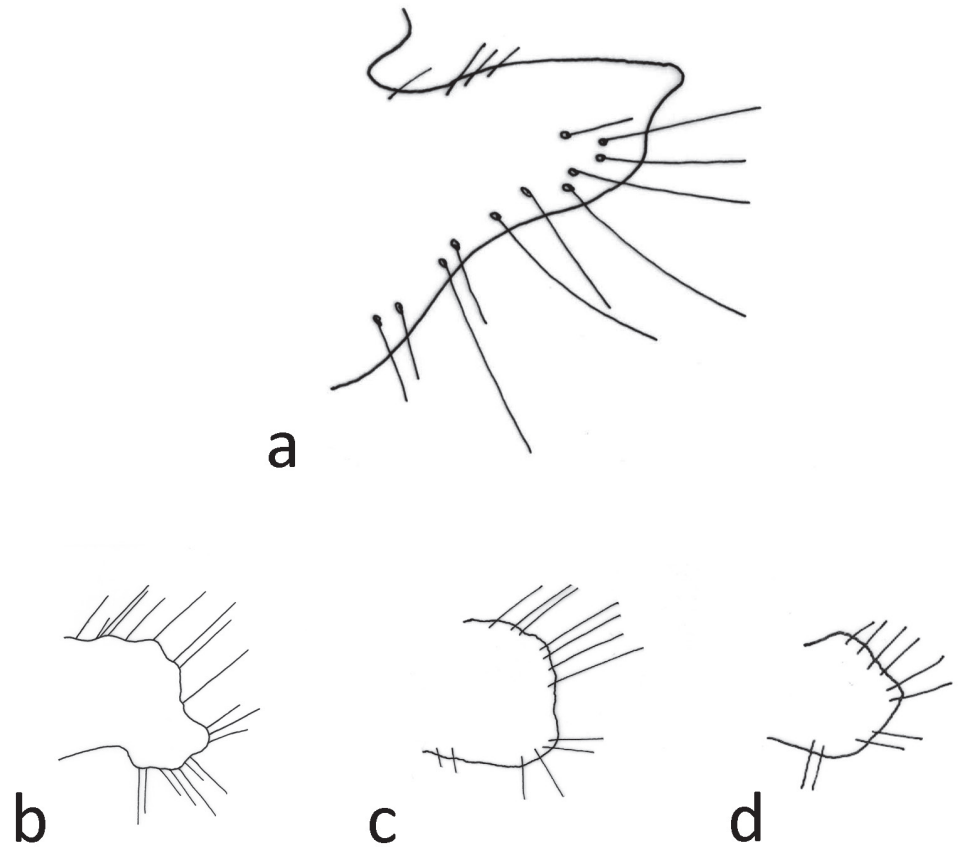

Figure 7. Chaetopteryx bucari sp. n., male genitalia, lateral view a inferior appendages b-d superior appendages.

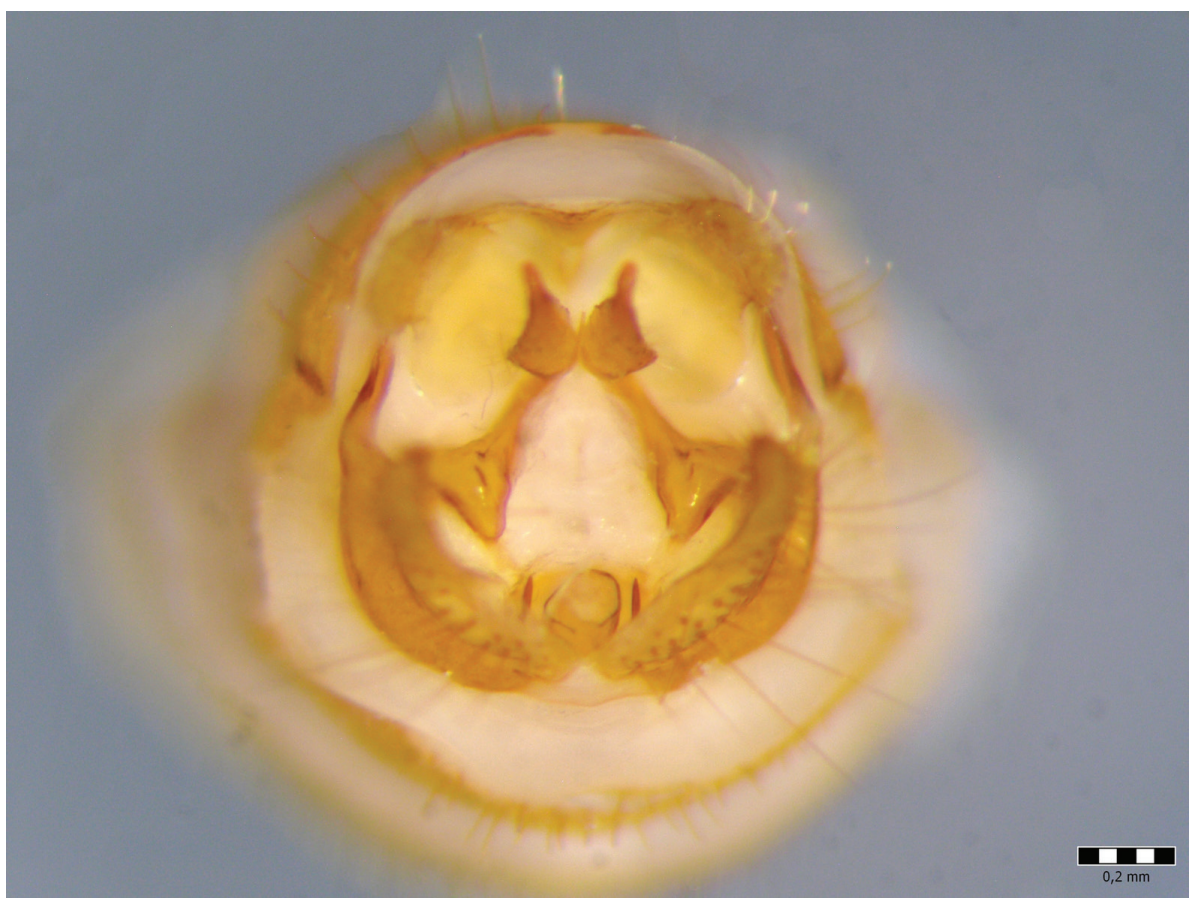

Figure 8. Chaetopteryx bucari sp. n., male genitalia, caudal view. 


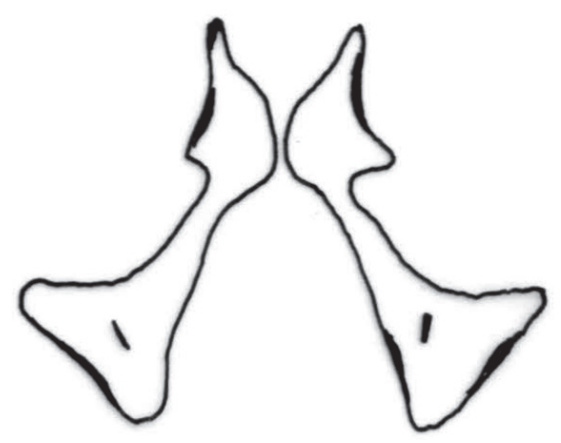

Figure 9. Chaetopteryx bucari sp. n., male genitalia, intermediate appendages (paraproctal complex), caudal view.

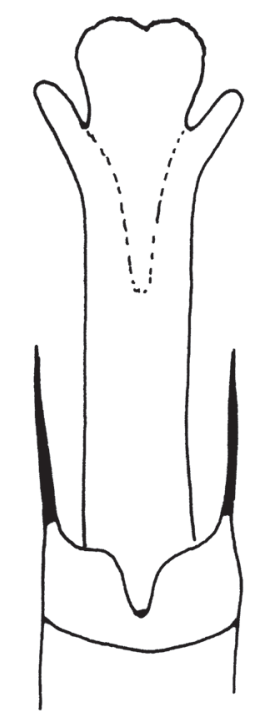

a
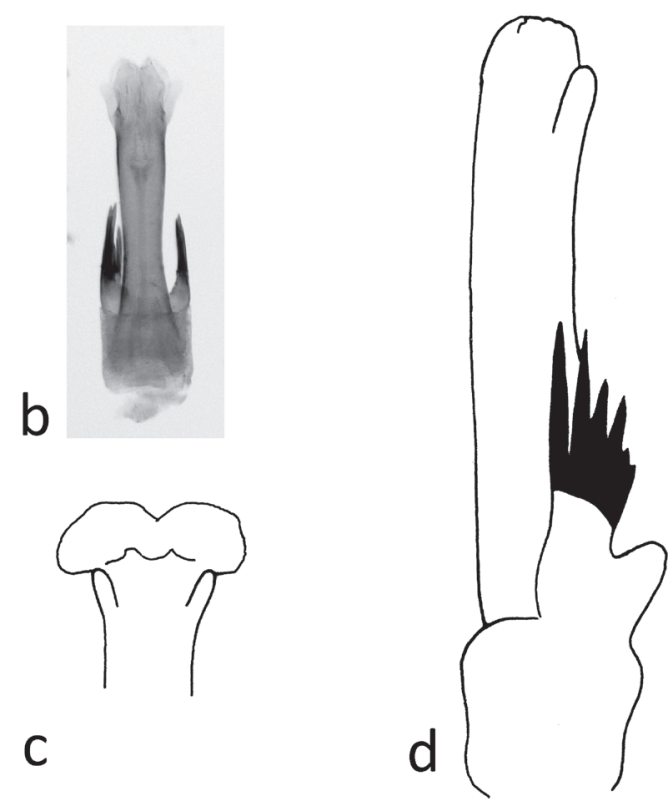

Figure 10. Chaetopteryx bucari sp. n., male genitalia, phallic organ (phallus): a dorsal view b ventral view c posterior membranous part of aedeagus $\mathbf{d}$ lateral view.

and small excision (recess) in middle (Figure 14). In ventral view anal tube broad with larger excision (recess) in middle than in dorsal side (Figure 15). Supragenital plate of segment $\mathrm{X}$ well-developed, triangular in shape in lateral and ventral views (Figures 12, 15). Lateral segment of vulvar scale relatively short in ventral view, with flat or slightly rounded apex (Figure 16a-c). Median lobe of vulvar scale (lower vulvar lip) with very small rounded or pointed apex (Figure 16b-d). In ca. 1/3rd of specimens' median lobe of vulvar scale not visible (Figure 16a).

Etymology. The species is dedicated to Professor Matija Bučar from the Faculty of Education, Department in Petrinja, University of Zagreb. 

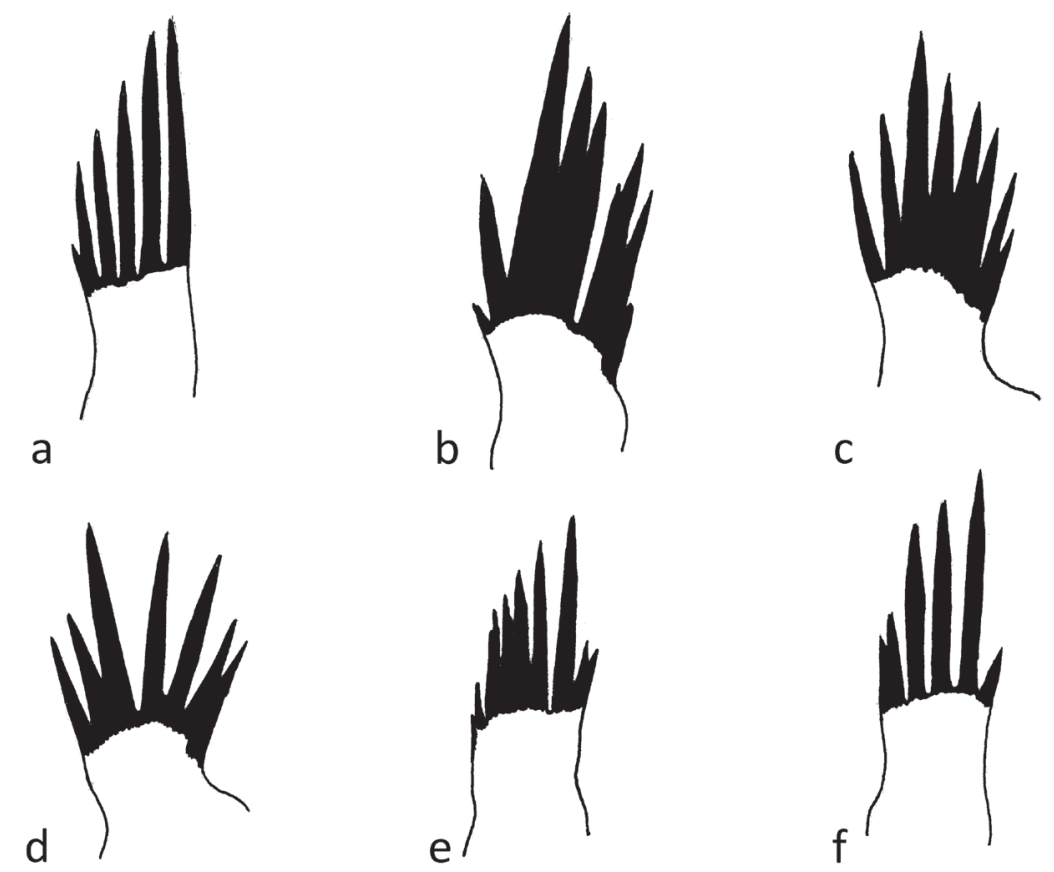

Figure II. Chaetopteryx bucari sp. n., male genitalia a-f parameres with sclerotized bristles.

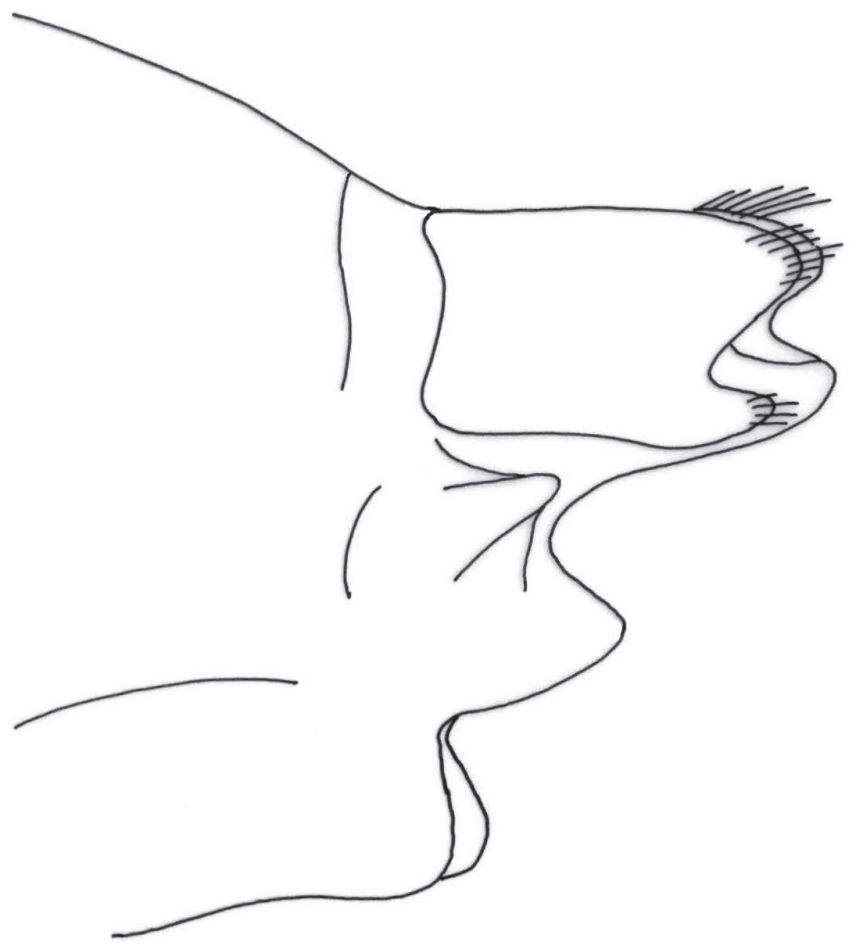

Figure 12. Chaetopteryx bucari sp. n., female genitalia, lateral view. 


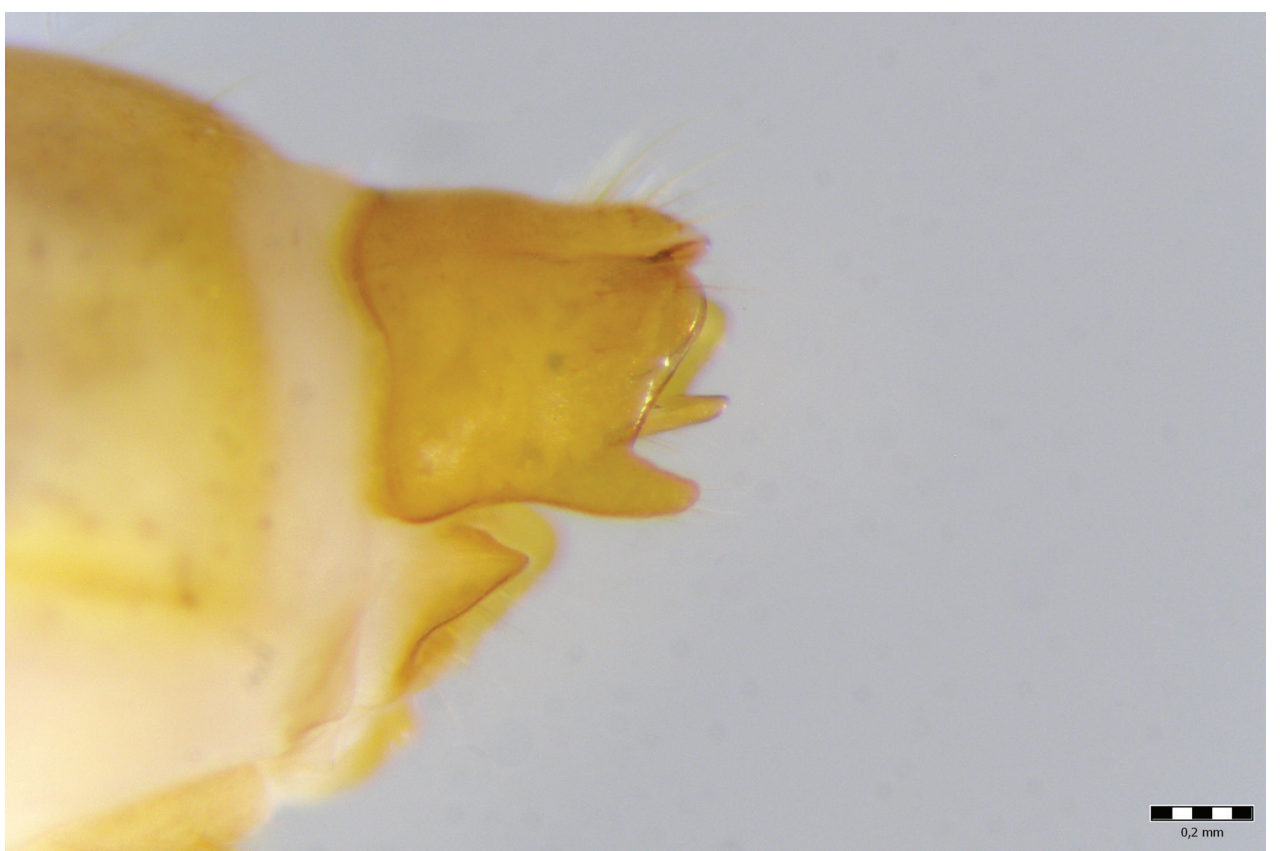

Figure I3. Chaetopteryx bucari sp. n., female genitalia, dorso-lateral view.

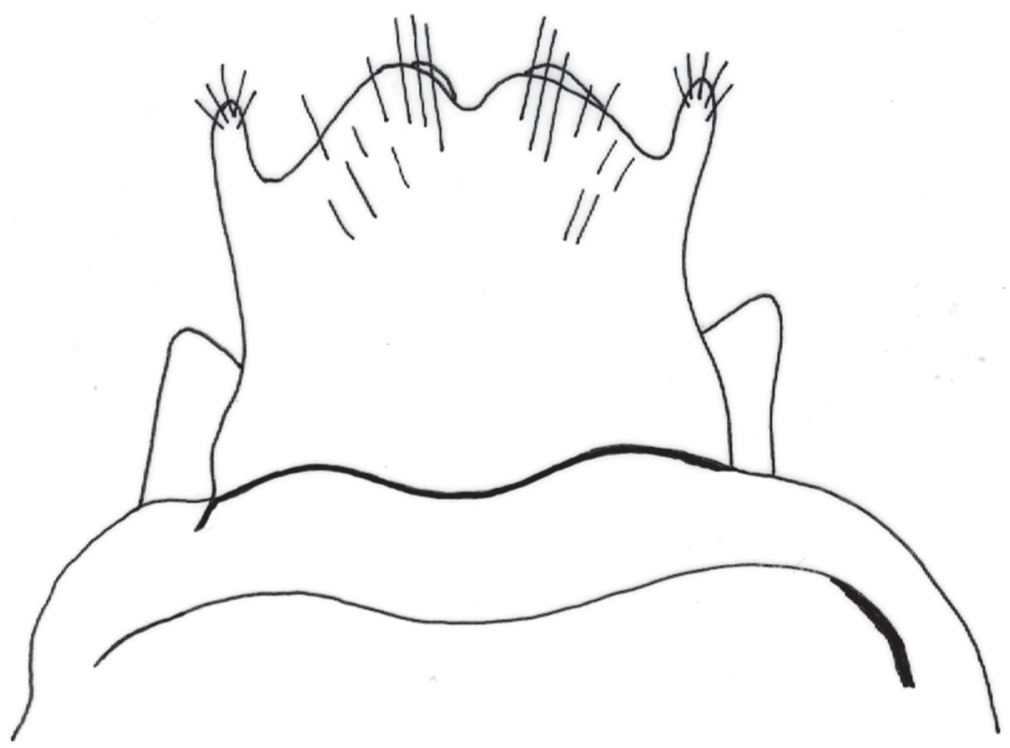

Figure I4. Chaetopteryx bucari sp. n., female genitalia, dorsal view.

Ecological notes and distribution. During our recent faunal surveys in Croatia and the Western Dinaric Balkan Chaetopteryx bucari was found only at 8 localities in the Banovina region (Table 1). The most distant sampling sites are $40 \mathrm{~km}$ apart (Slabi- 


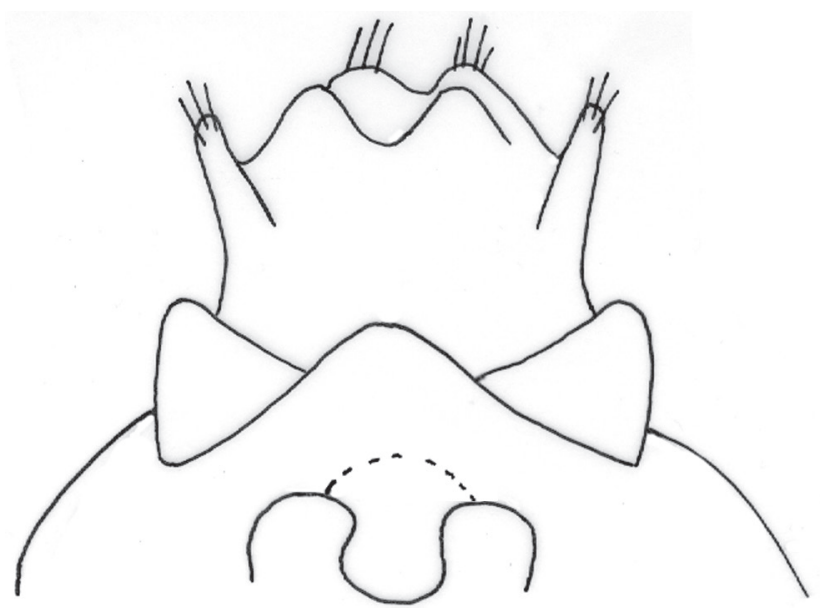

Figure 15. Chaetopteryx bucari sp. n., female genitalia, ventral view.

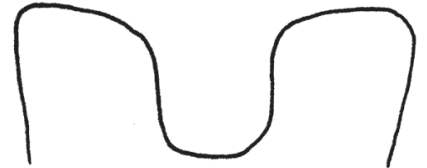

a

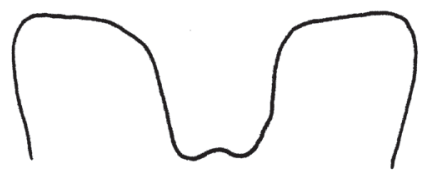

b
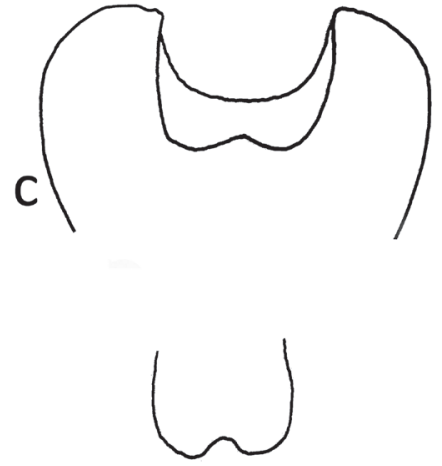

d

Figure 16. Chaetopteryx bucari sp. n., female genitalia a-d vulvar scale and median lobe of vulvar scale, ventral view.

nja and Gore). We collected C. bucari from 2 springs, 5 wellsprings and 1 location in the stream (Table 1). In total, we collected more than 580 specimens of C. bucari ( $85 \%$ were collected in pyramid-type emergence traps). The most abundant populations were found at Pecki spring and a headwater stream in Hrvatski Ćuntić. Over 150 specimens of C. bucari were observed on the night of October 14, 2010 on the walls of a small building next to the stream in Hrvatski Čuntić. In Pecki spring more than 50 specimens were observed on the night of October 31,2010. Chaetopteryx bucari was recorded at low altitudes between 104-185 m a.s.l. (Table 1).

Chaetopteryx bucari was collected in pyramid-type emergence traps from the end of September-December. The highest number of specimens was collected in October and November in both years. The sex ratio in both years was biased toward males,

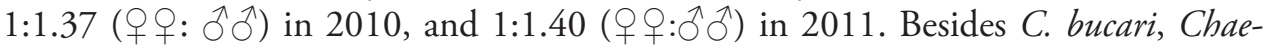


topteryx gonospina Marinković-Gospodnetić, 1966 and 2 additional caddisfly species (Limnephilus rhombicus (Linnaeus, 1758), Potamophylax pallidus Klapálek, 1898) were recorded in the emergence traps.

In addition to $C$. bucari 2 other species of the $C$. rugulosa group were collected in Croatia during our recent surveys. Chaetopteryx marinkovicae was collected from its type locality on the stream and spring in Kompanj village (Istria region); C. r. rugulosa was caught on Mt. Žumberak and Mt. Medvednica (northeast and central Croatia). Other species of Chaetopteryx found during this investigation were Chaetopteryx bosniaca Marinković-Gospodnetić, 1959 (Lika region), Chaetopteryx gonospina MarinkovićGospodnetić, 1966 (Banovina region), C. fusca (central Croatia, Dalmatia and Lika regions), and C. major (central Croatia).

\section{Discussion}

Systematic and taxonomic implications. Based on molecular evidence, we could confirm the hypothesis that Chaetopteryx bucari is a distinct species. Although C. bucari does not have a pp $>0.95$, it represents the sister taxon ( $\mathrm{pp}>0.95)$ to the highly supported C. r. schmidi. Furthermore, the mean genetic distance $(2.02 \%)$ between C. bucari and C. r. schmidi barely reached the $2-3 \%$ divergence observed as an interspecific genetic divergence in mtCOI sequences among some well-defined caddisfly species (Bálint et al. 2009, Pauls et al. 2009, Kučinić et al. 2011). However, among other well-defined caddisfly species this value can reach much higher levels (e.g., Zhou et al. 2007, Pauls et al. 2010), but also much lower values (e.g., Waringer et al. 2007). Thus reliance on distance methods alone for defining species boundaries is not advisable and species boundaries should be supported by additional lines of evidence such as additional, independent genes, morphology, or other independent characteristics (Zhou et al. 2007), particularly in taxa where hybridization is possible as is the case in Chaetopteryx (Malicky et al. 1986, Malicky and Pauls 2012). In the present study the genetic distinctiveness of $C$. bucari in combination with differences in morphological characters compared to its congeners, provide strong evidence to justify describing it as a new species.

In both sexes, especially in the adult female, $C$. bucari is relatively easily distinguishable from other taxa of the $C$. rugulosa group. The genetic data also show that specimens from 7 populations across the known range of the species from a clearly distinct clade from all other analysed Chaetopteryx. It is interesting that the female of $C$. bucari is particularly informative in diagnosing the species. In caddisflies this is quite unusual as males are generally more easily distinguished and females are often very difficult to differentiate from one another.

Based on the phylogenetic position of C.r. schmidi in relation to C. r. rugulosa and the other $C$. rugulosa subspecies, $C$. r. schmidi is well-defined and quite divergent from other members of the $C$. rugulosa clade based on molecular data. Thus, the subspecies C. $r$. schmidi is here re-established as a distinct species, C. schmidi, as it was described 
originally by Botosaneanu (1957) (Table 2) and not recognized as a subspecies of $C$. rugulosa as proposed by Malicky $(2004,2005)$.

Ecology. The emergence pattern of C. bucari corresponds with the general autumnal emergence patterns of the genus, usually from September-December, though emergence can be prolonged through January for some Chaetopteryx species (Kučinić 2002), including C. bucari (some specimens were collected by handpicking during January 2011). The emergence data from 2 years revealed that the sex ratio of $C$. bucari at the spring of Pecki stream is not exactly 1:1, but biased towards a surplus of males. In other studies applying the same methodology only a few species had 1:1 sex ratios (Kučinić 2002). In some species the sex ratio was 1:6 in favour of females (Previšić et al. 2007) and in other species males were dominant (Kučinić 2002, Semnički et al. 2011). These results are influenced by biological features of the species (e.g., emergence, oviposition behaviour of females), but may also be affected by trapping method (e.g., types of emergence pyramid-traps) (Malicky 2002).

Research on the diversity of large karst springs on the Balkan Peninsula has revealed high levels of caddisfly diversity. In some cases more than 20 species were collected from a single spring (Marinković-Gospodnetić 1979, Kučinić et al. 2008). This high alpha diversity of large karst springs does not, however, diminish the faunal significance of smaller springs. These are usually characterized by a small number of species, but often these species are highly specialized or local endemic species, such as $C$. bucari at the Pecki spring.

Distribution of Chaetopteryx rugulosa group in Croatia. At present, the genus Chaetopteryx is represented by 9 taxa in Croatia (Marinković-Gospodnetić 1979, Malicky and Krušnik 1988, Malicky 1996, 2004, Kučinić 2002, Kučinić et al. 2010, Previšić and Popijač 2010, Oláh 2010, 2011a). Including the new species C. bucari, 4 species from the Chaetopteryx rugulosa group (Malicky and Krušnik 1988, Malicky 1996, 2004, Oláh 2010) are now known from Croatia (Figure 17). Rare species from the genus Chaetopteryx are Chaetopteryx uherkovichi Oláh, 2011 distributed in eastern Croatia (Slavonia region) so far recorded only at the type locality (Oláh 2011a), C.r. mecsekensis known from only 1 locality in Croatia (Malicky 1996, 2004, Oláh 2010), but also distributed in Hungary (Malicky et al. 1986, Malicky 2004) and Serbia (Oláh 2010), and C. marinkovicae established in 3 localities in Istria (Malicky and Krušnik 1988). Our research did not confirm the presence of the latter species in 2 of these localities (Malicky and Krušnik 1988), but found specimens at the type locality in Kompanj village. Chaetopteryx marinkovicae is also known from Slovenia (Urbanič 2004).

Until now, the new species C. bucari was found only in the Banovina region, which is situated between rivers Sava and Kupa to the north and the state border with Bosnia and Herzegovina to the south and east (Figure 17). The Banovina region is characterised by rolling hills up to $600 \mathrm{~m}$ a.s.l. There are many small springs and streams in the region, and 3 large rivers, Una, Kupa and Sava, that form the border of the region. It is possible that $C$. bucari is also distributed in some other parts of continental Croatia or in Bosnia and Herzegovina, because we found this species in the valley of the Una River (Slabinja spring, Varoški bunar spring), which forms the border between these 2 countries. 


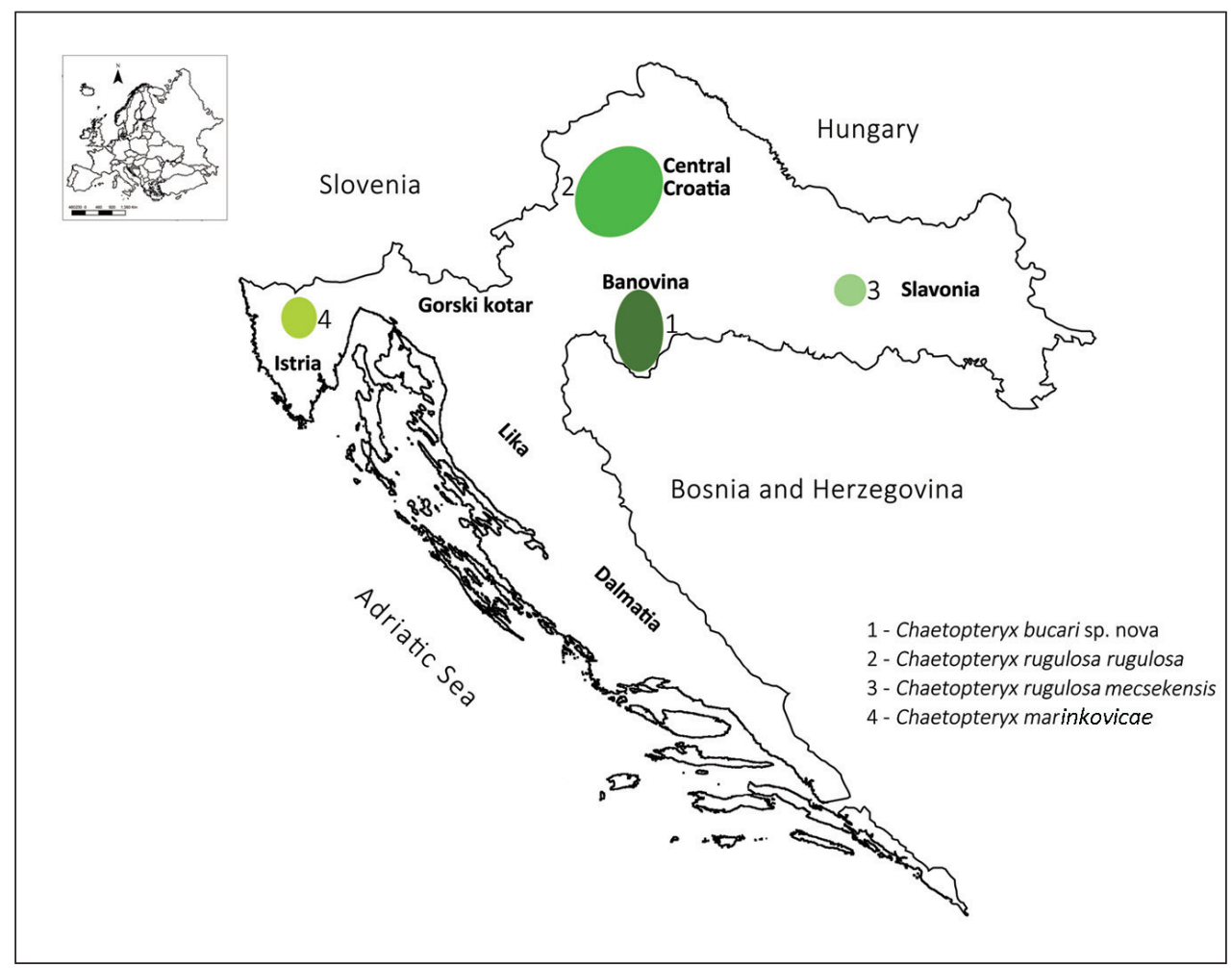

Figure 17. Distribution of Chaetopteryx rugulosa group in Croatia.

According to the current findings, C. bucari is not rare in the Croatian fauna. In fact, it is one of the most dominant caddisflies in the Banovina region. Along with $C$. fusca (Kučinić 2002, Semnički et al. 2011, Cerjanec 2012, M. Kučinić unpublished data) it is one of the most frequently found species from genus Chaetopteryx in Croatia. C. bucari inhabits springs and headwaters of small streams. The only known larger limnocrene spring that $C$. bucari inhabits is the Pašino vrelo spring.

Taxa from the C. rugulosa group have allopatric distributions in Croatia (Figure 17): C. bucari is distributed in the Banovina region, C. r. rugulosa in northern Croatia on Mt. Medvednica and Mt. Žumberak, C. r. mecsekensis in eastern Croatia on Mt. Papuk and C. marinkovicae in the sub-Mediterranean part of Croatia in Istria (Malicky and Krušnik 1988, Malicky 1996, 2004, Oláh 2010). Systematic research in mountain areas in Lika and Gorski kotar (Kučinić 2002, Kučinić et al. 2008, Previšić and Popijač 2010, Cerjanec 2012, Semnički et al. 2011, 2012) and the Mediterranean part of Croatia (Dalmatia region) (Graf et al. 2008, Waringer et al. 2009, Vučković 2011, Vučković et al. 2011, M. Kučinić unpublished data) did not result in collections of $C$. rugulosa group species in these areas.

Many members of the genus Chaetopteryx are either small-scale endemics or species with a low number of disjunct populations. This makes the group very interesting for biogeographic studies. There are several reasons that could explain the observed pattern 
of distribution: small populations, poor mobility of the winter emerging adults, and distribution in springs and in headwater reaches of small streams. Besides naturally isolating individual populations from one another, these aspects can also cause difficulties for investigating the genus, as it is hard to access many of the sites, especially in winter. Future investigations of this genus will be focused on poorly researched areas in Croatia and the western Balkans to gain a better understanding of the distribution and biogeography of Chaetopteryx in the region.

\section{Acknowledgements}

We express our gratitude to Xin Zhou for handling the mitochondrial barcoding of our specimens within the Barcoding of Life initiative. We thank Karl Kjer for sharing the previously unpublished "outgroup" data from BOLD. We thank two anonymous reviewers and Ralph Holzenthal for their valuable comments that helped improve the manuscript. For technical help we acknowledge senior curator Iva Mihoci, PhD, from the Croatian Natural Museum in Zagreb, Nediljko Landeka form the Institute of Public Health in Pula, and Milivoj Franjević from the Foresty Faculty, University of Zagreb. ISz was partially funded through an incoming research grant of the Biodiversity and Climate Research Centre in the frame of the research funding program LandesOffensive zur Entwicklung Wissenschaftlich-ökonomischer Exzellenz (LOEWE) of Hesse's Ministry of Higher Education, Research, and the Arts, Germany. SUP and MB acknowledge financial support from the research fundprogram "LOEWE - LandesOffensive zur Entwicklung Wissenschaftlich-ökonomischer Exzellenz" of Hesse's Ministry of Higher Education, Research, and the Arts. This research was supported by the Austrian Science Fund (FWF) as a part of Project P 23687-B17 and Croatian Ministry of Science, Education and Sports as a part of Project No. 119-1193080-1206.

\section{References}

Bálint M, Botosaneanu L, Ujvárosi L, Popescu O (2009) Taxonomic revision of Rhyacophila aquitanica (Trichoptera: Rhyacophilidae) based on molecular and morphological evidence and change of taxon status of Rhyacophila aquitanica ssp. carpathica to Rhyacophila carpathica sp. n. Zootaxa 2148: 39-48.

Botosaneanu L (1957) Quelques Trichopteres nouveaux de Roumanie. Tijdschrift voor entomologie 100: 179-194.

Botosaneanu L, Giudicelli J (2004) Contributions to the knowledge of the fauna of caddisflies (Insecta: Trichoptera) from south-east France, with description of new taxa. Annales de Limnologie - International Journal of Limnology 40: 15-32. doi: 10.1051/limn/2004002

Buckley T-R, Arensburger P, Simon C, Chambers G (2002) Combined data, Bayesian phylogenetics, and the origin of the New Zealand Cicada genera. Systematic Biology 51: 4-18. doi: $10.1080 / 106351502753475844$ 
Cerjanec D (2012) Ecological and biogeographical features of caddisflies (Insecta: Trichoptera) in different types of habitats in drainage of the Dobra River. PhD thesis (on Croatian), Zagreb, Croatia: University of Zagreb.

de Waard JR, Ivanova NV, Hajibabaei M, Hebert PDN (2008) Assembling DNA barcodes: analytical protocols. In: Martin CC (Ed) Environmental Genomics, Methods in Molecular Biology. Volume 410. Humana Press, Totowa, New Jersey, -275-283.

Drummond AJ, Ashton B, Buxton S, Cheung M, Cooper A, Duran C, Field M, Heled J, Kearse M, Markowitz S, Moir R, Stones-Havas S, Sturrock S, Thierer T, Wilson A (2011) Geneious v5.4, http://www.geneious.com/

Folmer O, Black M, Hoeh W, Lutz R, Vrijenhoek R (1994) DNA primers for amplification of mitochondrial cytochrome c oxidase subunit I from diverse metazoan invertebrates. Molecular Marine Biology and Biotechnology 3: 294-299.

Graf W, Kučinić M, Previšić A, Vučković I, Waringer J (2008) The larva, ecology and distribution of Tinodes braueri McLachlan, 1878 (Trichoptera: Psychomyiidae). Aquatic Insects 30 (4): 295-299. doi: 10.1080/01650420802331158

Guindon S, Gascuel O (2003) A simple, fast and accurate method to estimate large phylogenies by maximum-likelihood. Systematic Biology 52: 696-704. doi: $10.1080 / 10635150390235520$

Hajibabaei M, Dewaard JR, Ivanova NV, Ratnasingham S, Dooh RT, Kirk SL, Macki PM, Hebert PDN (2005) Critical factors for assembling a high volume of DNA barcodes. Philosophical Transactions of the Royal Society of London Series B: Biological Sciences 360: 1959-1967. doi: 10.1098/rstb.2005.1727

Hajibabaei M, Janzen DH, Burns JM, Hallwachs W, Hebert PDN (2006) DNA barcodes distinguish species of tropical Lepidoptera. Proceedings of the National Academy of Sciences of the United States of America 103: 968-971. doi: 10.1073/pnas.0510466103

Holzenthal RW, Blahnik RJ, Prather AL, Kjer KM (2007) Order Trichoptera Kirby, 1813 (Insecta), Caddisflies. Zootaxa 1668: 639-698.

Ivanova NV, Dewaard JR, Hebert PDN (2006) An inexpensive, automation-friendly protocol for recovering high-quality DNA. Molecular Ecology Notes 6: 998-1002. doi: 10.1111/j.1471-8286.2006.01428.x

Ivković M, Mičetić Stanković V, Mihaljević Z (2011) Emergence patterns and microhabitat preference of aquatic dance flies (Empididae; Clinocerinae and Hemerodromiinae) on a longitudinal gradient of barrage lake system. Limnologica 42 (1): 43-49. doi: 10.1016/j.limno.2011.07.003

Kučinić M (2002) Diversity and distribution of caddisflies in Plitvička Lakes. PhD thesis (on Croatian), Zagreb, Croatia: University of Zagreb.

Kučinić M, Bučar M, Delić A (2010) Prvi prilog poznavanju faune tulara (Insecta, Trichoptera) na području Banovine. In: Bučar M (Ed) Zbornik radova: Zrinska gora: regionalni park prirode. Matica hrvatska: 226-235.

Kučinić M, Previšić A, Gottstein A, Hrašovec B, Stanić-Koštroman S, Pernek M, Delić A (2008) Description of the larvae of Drusus radovanovici septentrionis Marinković-Gospodnetić, 1976 and Drusus croaticus Marinković-Gospodnetić, 1971 (Trichoptera: Limnephilidae) from Bosnia and Herzegovina and Croatia. Zootaxa 1783: 1-17. 
Kučinić M, Previšić A, Graf W, Jelaska L S, Stanić-Koštroman S, Waringer J (2011) Larval description, genetic and ecological features of Drusus radovanovici radovanovici MarinkovićGospodnetić, 1971 (Trichoptera, Limnephilidae) with some phylogenetic and taxonomic data on the bosnicus group in the Balkan Peninsula. Deutsche Entomologische Zeitschrift 58 (1): 135-153. doi: 10.1002/mmnd.201100010

Lodovici O, Valle M (2007) New data on the genus Chaetopteryx in Northern Italy and a description of $C$. morettii sp.n. Braueria 34: 15-16.

Malm T, Johanson KA (2011) A new classification of the long-horned caddisflies (Trichoptera: Leptoceridae) based on molecular data. BMC Evolutionary Biology 11: 10. doi: 10.1186/1471-2148-11-10

Malicky H (1996) Das Problem der allopatrischen Arten bei europäischen Köcherfliegen (Insecta: Trichoptera). Natura Croatica 5 (1): 11-23.

Malicky H (2002) A quantitative field comparasion of different types of emergence traps in a stream: general, Trichoptera, Diptera (Lemoniidae and Empididae). Annales de Limnologie - International Journal of Limnology 38: 133-149. doi: 10.1051/limn/2002011

Malicky H (2004) Atlas of European Trichoptera. Springer, Dordrecht, 359 pp.

Malicky H (2005) Ein kommentiertes Verzeichnis der Köcherfliegen (Trichoptera) Europas und des Mediterrangebietes. Linzer biologische Beiträge 37(1): 533-596.

Malicky H, Krušnik C (1988) Chaetopteryx marinkovicae sp.n. (Trichoptera, Limnephilidae) from Istria, Yugoslavia. Aquatic Insects 11 (3): 180. doi: 10.1080/01650428909361366

Malicky H, Krušnik C, Moretti G, Nógrádi S (1986) Ein Beitrag zur Kenntnis der Chaetopteryx rugulosa Kolenati, 1848 - Gruppe (Trichoptera, Limnephilidae). Entomofauna 7 (1): 1-27.

Malicky H, Pauls SU (2012) Cross-breeding of Chaetopteryx morettii and related species, with molecular and eidonomical results (Trichoptera, Limnephilidae). Annales de Limnologie International Journal of Limnology 48: 13-19. doi: 10.1051/limn/2011054

Marinković-Gospodnetić M (1979) Trichoptera (Insecta) velikih karstnih izvora u Dinaridima. In: Rauš (Ed) Drugi kongres Ekologa Jugoslavije (Second Congress of Ecologists of Yugoslavia). Savez društava ekologa Jugoslavije, Zagreb: 1837-1849.

Morse JC (Ed) (2013) Trichoptera World Checklist. http://entweb.clemson.edu/database/ trichopt/index.htm [accessed 26 May 2013]

Oláh J (2010) New species and new records of Palearctic Trichoptera in the material of the Hungarian Natural History Museum. Annales Historico-Naturales Musei Nationalis Hungarici 102: 65-117.

Oláh J (2011a) New species and records of Balkan Trichoptera. Folia Historico Naturalia Musei Matraensis 35: 111-121.

Oláh J (2011b) A new species of Chaetopteryx (Trichoptera, Limnephilidae) from the Northeast Carpathians. Braueria 38: 9-10.

Pauls SU, Blahnik RJ, Zhou X, Wardwell TC, Holzenthal RW (2010) DNA barcode data confirm new species and reveal cryptic diversity in Chilean Smicridea (Smicridea) (Trichoptera: Hydropsychidae). Journal of the North American Benthological Society 29: 1058-1074. doi: 10.1899/09-108.1

Pauls SU, Theissinger K, Ujvarosi L, Balint, M., Haase P (2009) Patterns of population structure in two closely related, partially sympatric caddisflies in Eastern Europe: historic intro- 
gression, limited dispersal, and cryptic diversity. Journal of the North American Benthological Society 28: 517-36. doi: 10.1899/08-100.1

Posada D (2008) jModelTest: Phylogenetic Model Averaging. Molecular Biology and Evolution 25: 1253-1256. doi: 10.1093/molbev/msn083

Previšić A, Kerovec M, Kučinić M (2007) Emergence and Composition of Trichoptera from Karst Habitans, Plitvice lakes Region, Croatia. International Review of Hydrobiology 92 (1): 61-83. doi: 10.1002/iroh.200510921

Previšić A, Popijač A (2010) Caddisfly (Insecta: Trichoptera) fauna of Kupa and Čabranka rivers and their tributaries, Gorski kotar, W Croatia. Natura Croatia 19 (2): 357-368.

Rambaut A, Drummond AJ (2009) Tracer Version 1.5, http://beast.bio.edsac.uk/Tracer

Ratnasingham S, Hebert PDN (2007) BOLD: the Barcode of Life Data System (www. barcodinglife.org). Molecular Ecology Notes 7: 355-364. doi: 10.1111/j.14718286.2007.01678.x

Rodríguez F, Oliver JL, Marin A, Medina JR (1990) The general stochastic model of nucleotide substitution. Journal of Theoretical Biology 142 (4): 484-501. doi: 10.1016/S00225193(05)80104-3

Ronquist F, Huelsenbeck JP (2003) MrBayes 3: Bayesian phylogenetic inference under mixed models. Bioinformatics 19: 1572-1574. doi: 10.1093/bioinformatics/btg180

Semnički P, Previšić A, Ivković M, Čmrlec K, Mihaljević Z (2011) Emergence of caddisfly (Trichoptera, Insecta) at tufa barriers in Plitvice Lakes National Park. Entomologia Croatica 115 (1-4): 145-161. doi: 10.1002/iroh.201101500

Semnički P, Previšić A, Ivković M, Čmrlec K, Mihaljević Z (2012) Tufa Barriers from a Caddisfly's Point of View: Streams or Lake Outlets? International Review of Hydrobiology 97 (6): 465-484.

Sipahiler F (2010) Two New Species of Chaetopteryx Stephens, 1837 from Turkey with a Description of the Unknown Female of C. bektasensis Sipahiler, 2008 (Trichoptera, Limnephilidae: Limnephilinae: Chaetopterygini). Psyche: A Journal of Entomology. doi: 10.1155/2010/180671

Tamura K, Peterson D, Peterson N, Stecher G, Nei M, Kumar S (2011) MEGA5: Molecular Evolutionary Genetics Analysis using Maximum Likelihood, Evolutionary Distance, and Maximum Parsimony Methods. Molecular Biology and Evolution 28: 2731-2739. doi: $10.1093 / \mathrm{molbev} / \mathrm{msr} 121$

Urbanič G (2004) Ecology and distribution of caddisflies (Insecta: Trichoptera) in some watercourses in Slovenia. PhD thesis (on Slovenian), Ljubljana, Slovenia: University of Ljubljana.

Urbanič G, Krušnik C (2003) The female of Chaetopteryx irenae Krušnik \& Malicky 1986. Acta Entomologica Slovenica 10 (1): 21-24.

Vučković I (2011) Faunal, taxonomical and ecological characters of caddisflies (Insecta, Trichoptera) drainage of the River Cetina. PhD thesis (on Croatian), Zagreb, Croatia: University of Zagreb. doi: 10.1080/01650424.2011.641181

Vučković I, Previšić A, Graf W, Kučinić M (2011) Description of the female and distribution of Annitella apfelbecki Klapálek, 1899 (Insecta: Trichoptera). Aquatic Insects 33 (4): 381-389. Waringer J, Graf W, Kučinić M, Previšić A, Vučković A (2009) The Larva and life cycle of Annitella apfelbecki Klápalek, 1899, including a re-description of Melampophylax nepos 
McLachlan, 1880 (Trichoptera: Limnephilidae). Aquatic Insects 31 (1): 71-80. doi: 10.1080/01650420802616327

Waringer J, Graf W, Pauls S, Lubini V (2007) The Larva of Drusus nigrescens Meyer-Dur, 1875 (Trichoptera: Limnephilidae: Drusinae) with notes on its ecology, genetic differentiation and systematic position. Annales de Limnologie - International Journal of Limnology 43: 161-166. doi: 10.1051/limn:2007010

Zhou X, Kjer KM, Morse JC (2007) Associating larvae and adults of Chinese Hydropsychidae caddisflies (Insecta: Trichoptera) using DNA sequences. Journal of the North American Benthological Society 26: 719-742. doi: 10.1899/06-089.1 